\title{
Design and Synthesis of Novel Oxime Ester Photoinitiators Augmented by Automated Machine Learning
}

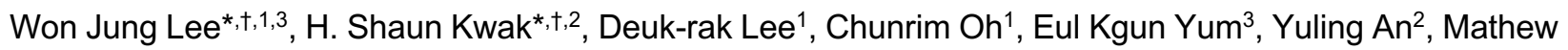
D. Halls ${ }^{2}$, and Chi-Wan Lee ${ }^{1}$

${ }^{1}$ Convergence Specialty Materials R\&D Center, Samyang Corp., Yuseong-gu, Daejeon, Korea

2 Schrödinger, Inc. New York, NY 10036, USA

${ }^{3}$ Department of Chemistry, Chungnam National University, Yuseong-gu, Daejeon 34134, Korea

† E-mail: wonjung.lee@samyang.com; shaun.kwak@schrodinger.com

* These authors contributed equally to this work

\section{SUPPORTING INFORMATION}

\section{Contents:}

1. Chemistry of photoinitiator candidates

Table S1. Chemical structures of the photoinitiator candidates.

\section{Details of experimental setups}

Figure S1. Absorption spectra of representative oxime ester (AN-31A, orange) and keto-oxime ester (AN-31B, green) photoinitiator candidates.

2.1. Preparation of binder resin

2.2. Preparation of photoresist compositions

2.3. Evaluation of photoresist compositions with photosensitivity

2.4. Photoreaction mechanisms

Figure S2. Illustrations of photoreaction mechanisms for (a) oxime ester type and (b) keto-oxime ester type photoinitiators synthesized and examined in this work.

Figure S3. Wavelength information of the lights emitted from the mask aligner.

3. Summary of performance data for machine learning models

Table S2. Confusion matrix elements from the top-five categorical models.

Figure S4. Scatter plots from the three best-performing continuous models.

4. Additional model visualization examples from the best-performing continuous model

Figure S5. Model visualization with chemical groups decreasing the photosensitivity.

Figure S6. Model visualization for the three newly designed photoinitiator candidates.

\section{Method of synthesis for the new photoinitiator candidates}

Scheme S1. Synthetic pathway for the newly-designed photoinitiator candidate 1.

Figure S7-S10. NMR data obtained during synthesis of photoinitiator candidate 1.

Scheme S2. Synthetic pathway for the newly-designed photoinitiator candidate 2.

Figure S11-S14. NMR data obtained during synthesis of photoinitiator candidate 2.

Scheme S3. Synthetic pathway for the newly-designed photoinitiator candidate 3.

Figure S15-S18. NMR data obtained during synthesis of photoinitiator candidate 3. 


\section{Chemistry of photoinitiator candidates}

Table S1. Chemical structures of the photoinitiator candidates represented by SMILES strings.

\begin{tabular}{|c|c|}
\hline Name & SMILES \\
\hline AN-21A & $\mathrm{CC}(=0) \mathrm{O} / \mathrm{N}=\mathrm{C}(\backslash \mathrm{C}) \mathrm{c}(\mathrm{cc} 1) \mathrm{c}(\mathrm{c} 2 \mathrm{c} 13) \operatorname{cccc} 2 \mathrm{CC} 3$ \\
\hline AN-31A & $\mathrm{CC}(=0) \mathrm{O} \backslash \mathrm{N}=\mathrm{C}(\mathrm{CC}) \backslash \mathrm{c}(\mathrm{cc} 1) \mathrm{c}(\mathrm{c} 2 \mathrm{c} 13) \mathrm{cccc} 2 \mathrm{CC} 3$ \\
\hline B-BP21B & $\mathrm{CC}(=0) \mathrm{O} / \mathrm{N}=\mathrm{C}(\backslash \mathrm{C}) \mathrm{C}(=0) \mathrm{c} 1 \mathrm{ccc}(\mathrm{cc} 1)-\mathrm{c} 2 \mathrm{ccc}(\mathrm{cc} 2) \mathrm{C}(=0) \mathrm{c} 3 \operatorname{ccccc} 3$ \\
\hline BI-P-31A & $\operatorname{c1cccc}(\operatorname{c12}) \mathrm{C}(=0) \mathrm{N}(\mathrm{C} 2=0) \mathrm{Cc} 3 \operatorname{ccc}(\mathrm{cc} 3) / \mathrm{C}(\mathrm{CC})=\mathrm{N} \backslash \mathrm{OC}(=0) \mathrm{C}$ \\
\hline BN14-61A & $\operatorname{cccccC}(=\mathrm{N} / \mathrm{OC}(=0) \mathrm{C}) \backslash \mathrm{c}(\mathrm{cc} 1) \mathrm{cc}(\mathrm{c} 12) \mathrm{nc}(\mathrm{C}) \mathrm{n} 2 \mathrm{CccC}$ \\
\hline BN14-61B & $\operatorname{ccccC}(=N / O C(=0) C) \backslash C(=0) c(\operatorname{cc} 1) \operatorname{cc}(c 12) n c(C) n 2 C c c C$ \\
\hline BP-N43 & $N \# C c 1 c c c(c c 1)-c 2 c c c(c c 2) / C(C)=N \backslash O C(=0) C$ \\
\hline BP-Ph1A & $\operatorname{c1ccccc} 1 \mathrm{C} / \mathrm{C}(=\mathrm{N} / \mathrm{OC}(=0) \mathrm{C}) \operatorname{c2ccc}(\operatorname{cc} 2)-\mathrm{c} 3 \operatorname{ccccc} 3$ \\
\hline BP-Ph21B & $\operatorname{c1ccccc} 1 / C(=N \backslash O C(=O) C) C(=0) \operatorname{coccc}(\operatorname{cc} 2)-\operatorname{coccccc} 3$ \\
\hline BPA-31-TM & $C C(=0) O / N=C(\backslash C(C)(C) C) c 1 c c c(c c 1)-c 2 \operatorname{ccccc} 2$ \\
\hline BPB-33B & $\operatorname{c1cc}([\mathrm{N}+](=0)[\mathrm{O}-]) \operatorname{ccc} 1-\mathrm{c} 2 \operatorname{ccc}(\mathrm{cc} 2) \mathrm{C}(=\mathrm{O}) / \mathrm{C}(\mathrm{C})=\mathrm{N} / \mathrm{OC}(=0) \mathrm{ccc}$ \\
\hline BPB-35B & $\operatorname{c1cc}([\mathrm{N}+](=0)[0-]) \operatorname{ccc} 1-\operatorname{coccc}(\operatorname{cc} 2) \mathrm{C}(=0) / \mathrm{C}(\mathrm{C})=\mathrm{N} / \mathrm{OC}(=0) \operatorname{ccccc}$ \\
\hline BPH-61A & $\operatorname{c1} 1 \operatorname{ccccc} 1-\mathrm{c} 2 \operatorname{ccc}(\mathrm{cc} 2) / \mathrm{C}(=\mathrm{N} / \mathrm{OC}(=0) \mathrm{C}) \mathrm{ccccc}$ \\
\hline BPH-NB1 & {$[0-][N+](=0) c 1 c c c(\operatorname{cc} 1) / C(=N \backslash O C(=0) C) \operatorname{coccc}(\operatorname{cc} 2)-c 3 \operatorname{ccccc} 3$} \\
\hline CAZ-102 & $\mathrm{O}=[\mathrm{N}+]([\mathrm{O}-]) \mathrm{c}(\mathrm{c} 1) \operatorname{ccc}(\mathrm{c} 12) \mathrm{n}(\mathrm{COCC}) \mathrm{c} 3 \mathrm{c} 2 \mathrm{cc}(\mathrm{cc} 3) \mathrm{C}(=\mathrm{O}) / \mathrm{C}(\mathrm{C})=\mathrm{N} \backslash \mathrm{OC}(=0) \mathrm{C}$ \\
\hline CAZ-N96 & $\mathrm{c} 1 \mathrm{cc}(\mathrm{OC}) \mathrm{cc}(\mathrm{c} 12) \mathrm{c} 3 \mathrm{c}(\mathrm{n} 2 \mathrm{CC}) \mathrm{ccc}(\mathrm{c} 3) \mathrm{C}(=\mathrm{O}) / \mathrm{C}(\mathrm{C})=\mathrm{N} \backslash \mathrm{OC}(=0) \mathrm{C}$ \\
\hline FL-N23 & $C C(=0) O / N=C(\backslash C(=0) C) C(=0) c(c 1) \operatorname{ccc}(c 12) c 3 c(\operatorname{cccc} 3) C 2(C C C C) c c C C$ \\
\hline FL-N25 & $C C(=0) 0 / N=C(\backslash C(=0) C) C(=0) c 1 c c c(c c 1)-c 2 c c c(B r) c c 2$ \\
\hline FL-N32 & $C C(=0) 0 / N=C(\backslash C(=0) C) C(=0) c(c c 1) c c(c 12) \operatorname{cccc} 2$ \\
\hline FL-N33 & $\operatorname{c1cccc}(\mathrm{c} 12) \operatorname{cc}(\operatorname{cc} 2) \mathrm{C}(=0) / \mathrm{C}(\mathrm{C}(=0) \mathrm{C})=\mathrm{N} / \mathrm{OC}(=0) \operatorname{coccccc} 3$ \\
\hline FL-N34 & $C C(=0) O / N=C(\backslash C(=0) C) C(=0) c(\operatorname{ccc} 1) c(c 12) \operatorname{cccc} 2$ \\
\hline FL-N37 & $\operatorname{c1} 1 \operatorname{ccccc} 1 \mathrm{C}(=0) 0 / \mathrm{N}=\mathrm{C}(\backslash \mathrm{C}(=0) \mathrm{C}) \mathrm{C}(=0) \operatorname{coc} \operatorname{ccc}(\operatorname{cc} 2)-\operatorname{coc} \operatorname{cccc} 3$ \\
\hline FL-N75 & $O C(=0) c 1 \operatorname{ccccc} 1 C(c(c 23) \operatorname{cccc} 2)=C 4[C @ @ H] 3 C=C(C=C 4) / C(C)=N / O C(=O) C$ \\
\hline IA-231 & $\mathrm{CC}(=0) \mathrm{O} / \mathrm{N}=\mathrm{C}(/ \mathrm{CCC}) \mathrm{c}(\mathrm{cn} 1 \mathrm{CC}) \mathrm{c}(\mathrm{c} 12) \operatorname{cccc} 2$ \\
\hline NP-N45 & $C C(=0) 0 / N=C(\backslash C C) c 1 c(0) c(c c(C) c 1)-n(n 2) n c(c 23) c c c c 3$ \\
\hline
\end{tabular}




\begin{tabular}{|c|c|}
\hline PTD-31B & $\mathrm{O}=\mathrm{C}(\mathrm{C}) \mathrm{O} \backslash \mathrm{N}=\mathrm{C}(\mathrm{C}) \backslash \mathrm{C}(=\mathrm{O}) \mathrm{c}(\mathrm{c} 1) \operatorname{ccc}(\mathrm{c} 1 \mathrm{Sc}(\mathrm{c} 23) \mathrm{cccc} 2) \mathrm{N} 3 \mathrm{CCCCCCCCCC}$ \\
\hline PTI-31B & $\mathrm{CC}(=0) \mathrm{O} / \mathrm{N}=\mathrm{C}(\backslash \mathrm{C}) \mathrm{C}(=0) \mathrm{c} 1 \mathrm{ccc}(\mathrm{cc} 1) \mathrm{CN}(\mathrm{C} 2=0) \mathrm{C}(=0) \mathrm{c}(\mathrm{c} 23) \operatorname{cccc} 3$ \\
\hline QBA-11 & $\mathrm{n} 1 \mathrm{cccc}(\mathrm{c} 12) \mathrm{c}(/ \mathrm{C}(\mathrm{C})=\mathrm{N} / \mathrm{OC}=\mathrm{O}) \mathrm{C}) \operatorname{ccc} 2 \mathrm{OCc} 3 \mathrm{ccccc} 3$ \\
\hline SOF-61A & $\operatorname{cccccc}(=N \backslash O C(=0) C) \backslash c(c c 1) \operatorname{cc}(c 12) C c 3 c 2 c c c(c 3) / C(=N / O C(=0) C) \operatorname{ccccC}$ \\
\hline SBF-21A & $\mathrm{CC}(=0) \mathrm{O} \backslash \mathrm{N}=\mathrm{C}(\mathrm{C}) \backslash \mathrm{c}(\mathrm{cc} 1[\mathrm{~N}+](=0)[0-]) \mathrm{cc}(\mathrm{c} 12) \mathrm{C}(\mathrm{CCCC})(\mathrm{CCCC}) \mathrm{c} 3 \mathrm{c} 2 \mathrm{ccc}(\mathrm{c} 3) / \mathrm{C}(\mathrm{C})=\mathrm{N} \backslash \mathrm{OC}(=0) \mathrm{C}$ \\
\hline SBF-31A & $C C(=0) O \backslash N=C(C C) \backslash c(c c 1) c c(c 12) C(C C C C)(C C C C) c 3 c 2 c c c(c 3) / C(C C)=N \backslash O C(=0) C$ \\
\hline SBF-41A & $C C(=0) O \backslash N=C(C C C) \backslash c(c c 1) c c(c 12) C(C C C C)(C C C C) c 3 c 2 c c c(c 3) / C(C C C)=N / O C(=0) C$ \\
\hline SBF-51A & $\operatorname{cCccC}(=N / O C(=0) C) \backslash c(\operatorname{cc} 1) c c(c 12) C(C C C C)(C C C C) c 3 c 2 c c c(c 3) / C(C C C C)=N / O C(=0) C$ \\
\hline SBF-51B & $\mathrm{O}=\mathrm{C}(\mathrm{C}) \mathrm{O} \backslash \mathrm{N}=\mathrm{C}(\mathrm{CCC}) \backslash \mathrm{C}(=\mathrm{O}) \mathrm{c}(\mathrm{cc} 1) \mathrm{cc}(\mathrm{c} 12) \mathrm{C}(\mathrm{CCCC})(\mathrm{CCCC}) \mathrm{c} 3 \mathrm{c} 2 \mathrm{ccc}(\mathrm{c} 3) \mathrm{C}(=0) / \mathrm{C}(\mathrm{CCC})=\mathrm{N} / \mathrm{OC}(=0) \mathrm{C}$ \\
\hline SBF-61B & $\operatorname{cccccC}(=N / O C(=0) C) \backslash c(\operatorname{cc} 1) \operatorname{cc}(\mathrm{c} 12) \mathrm{C}(\mathrm{CCCC})(\mathrm{CCCC}) \mathrm{c} 3 \mathrm{c} 2 \mathrm{ccc}(\mathrm{c} 3) / \mathrm{C}(=\mathrm{N} / \mathrm{OC}(=0) \mathrm{C}) \mathrm{CcccC}$ \\
\hline TP-N47 & $C C(=0) O / N=C(\backslash C) C(=0) c 1 c(O C(=0) C) c(c c(C) c 1)-n(n 2) n c(c 23) c c c c 3$ \\
\hline TP-N48 & $C C(=0) 0 / N=C(\backslash C) C(=0) c 1 c(0) c(c c(C) c 1)-n(n 2) n c(c 23) c c c c 3$ \\
\hline TPA-N81 & $C C(=0) O \backslash N=C(C) \backslash C(=0) c(\operatorname{cc} 1) \operatorname{ccc} 1 N(\operatorname{coccc}(\operatorname{cc} 2) O C) \operatorname{coccc}(\operatorname{cc} 3) C(=0) / C(C)=N / O C(=0) C$ \\
\hline AN-31B & $\mathrm{CC}(=0) \mathrm{O} / \mathrm{N}=\mathrm{C}(\backslash \mathrm{C}) \mathrm{C}(=0) \mathrm{c}(\mathrm{cc} 1) \mathrm{c}(\mathrm{c} 2 \mathrm{c} 13) \operatorname{cccc} 2 \mathrm{CC} 3$ \\
\hline BI-MPH-31B & $C C(=0) O \backslash N=C(C) \backslash C(=0) c(c c 1) c c(c 12) n c(n 2 C C C C)-c 3 c c c c c 3$ \\
\hline $\mathrm{BN}-21 \mathrm{~B}$ & $\mathrm{CC}(=0) \mathrm{O} / \mathrm{N}=\mathrm{C}(/ \mathrm{C}) \mathrm{C}(=0) c(\mathrm{c}(\mathrm{c} 12) \operatorname{ccc} 2) \operatorname{ccc} 1-\mathrm{c} 3 \operatorname{ccc}(\mathrm{c}(\mathrm{c} 34) \operatorname{cccc} 4) \mathrm{C}(=0) / \mathrm{C}(\mathrm{C})=\mathrm{N} / \mathrm{OC}(=0) \mathrm{C}$ \\
\hline BP-31B & $\mathrm{CC}(=0) \mathrm{O} / \mathrm{N}=\mathrm{C}(\backslash \mathrm{C}) \mathrm{C}(=0) \mathrm{c} 1 \mathrm{ccc}(\mathrm{cc} 1)-\mathrm{c} 2 \mathrm{ccccc} 2$ \\
\hline BP-62A & $\mathrm{c} 1 \mathrm{ccccc} 1-\mathrm{c} 2 \mathrm{ccc}(\mathrm{cc} 2) / \mathrm{C}(\mathrm{CCCCC})=\mathrm{N} / \mathrm{OC}(=\mathrm{O}) \mathrm{CC}$ \\
\hline CAZ-N69 & $\mathrm{CC}(=0) \mathrm{O} / \mathrm{N}=\mathrm{C}(\backslash \mathrm{C}) \mathrm{C}(=0) \mathrm{c} 1 \mathrm{ccc}(\mathrm{cc} 1) \mathrm{N}(\mathrm{c} 2 \mathrm{ccccc} 2) \mathrm{c} 3 \mathrm{ccccc} 3$ \\
\hline CAZ-N76 & $C C(=0) O / N=C(\backslash C) C(=0) c 1 c c c(\operatorname{cc} 1) N(\operatorname{coccccc} 2) \operatorname{coccc}(\operatorname{cc} 3) C(=0) / C(C)=N / O C(=0) C$ \\
\hline CAZ-N79 & $c 1 c c(O C) c c c 1-n(c(c 23) \operatorname{ccc} 2) c 4 c 3 c c(c c 4) C(=0) / C(C)=N \backslash O C(=0) c$ \\
\hline CAZ-N85 & $O=C(C) O \backslash N=C(C) \backslash C(=0) c(c 1) c c c(c 12) n(-c 3 c c c(c c 3) O C) c 4 c 2 c c(c c 4) C(=0) / C(C)=N \backslash O C(=0) C$ \\
\hline CAZ-N90 & $c 1 c c(O C) c c c 1-n(c(c 23) c c c(c 2) O C) c 4 c 3 c c(c c 4) C(=0) / c(C)=N \backslash O C(=0) C$ \\
\hline CAZ-N92 & $\mathrm{CC}(=0) \mathrm{O} / \mathrm{N}=\mathrm{C}(\backslash \mathrm{C}) \mathrm{C}(=0) \mathrm{c}(\mathrm{cc} 1) \mathrm{cc}(\mathrm{c} 12) \mathrm{c} 3 \mathrm{c}(\mathrm{ccc}(\mathrm{c} 3) \mathrm{OC}) \mathrm{n} 2-\mathrm{c} 4 \mathrm{ccc}(\mathrm{cc} 4) \mathrm{SC}$ \\
\hline CAZ-N94 & $\mathrm{CC}(=0) 0 / \mathrm{N}=\mathrm{C}(\backslash \mathrm{C}) \mathrm{C}(=0) \mathrm{c}(\mathrm{cc} 1) \mathrm{cc}(\mathrm{c} 12) \mathrm{c} 3 \mathrm{c}(\mathrm{n} 2 \mathrm{CC}) \mathrm{cccc} 3$ \\
\hline DB-3P1B & $C C(=0) O \backslash N=C(C) \backslash C(=0) c(c c 1) c c(c 12) C(C C C C)(C C C C) c 3 c 2 c c c(c 3) C(=0) / C(=N \backslash O C(=0) C) c 4 c c c c c 4$ \\
\hline DBF-31B & $\operatorname{c1cccc}(\mathrm{c} 12) \mathrm{C}(\mathrm{CCCC})(\mathrm{CCCC}) \mathrm{c} 3 \mathrm{c} 2 \mathrm{ccc}(\mathrm{c} 3) \mathrm{C}(=\mathrm{O}) / \mathrm{C}(\mathrm{C})=\mathrm{N} / \mathrm{OC}(=0) \mathrm{C}$ \\
\hline DBF-32B & $\mathrm{CCC}(=0) \mathrm{O} / \mathrm{N}=\mathrm{C}(/ \mathrm{C}) \mathrm{C}(=0) \mathrm{c}(\mathrm{c} 1) \operatorname{ccc}(\mathrm{c} 12) \mathrm{c} 3 \mathrm{c}(\mathrm{cccc} 3) \mathrm{C} 2(\mathrm{CCCC}) \mathrm{CCCC}$ \\
\hline DEF-41B & $C C(=0) O / N=C(/ C C) C(=0) c(c 1) \operatorname{ccc}(c 12) c 3 c(C 2(C C) C C) c c c c 3$ \\
\hline
\end{tabular}




\begin{tabular}{|c|c|}
\hline DEF-42B & $\mathrm{CCC}(=0) 0 / \mathrm{N}=\mathrm{C}(/ \mathrm{CC}) \mathrm{C}(=0) \mathrm{c}(\mathrm{c} 1) \mathrm{ccc}(\mathrm{c} 12) \mathrm{c} 3 \mathrm{c}(\mathrm{C} 2(\mathrm{CC}) \mathrm{CC}) \mathrm{cccc} 3$ \\
\hline DEF-52B & $c 1 c c c c(c 12) C(C C)(C C) c 3 c 2 c c c(c 3) C(=0) / C(C C C)=N \backslash O C(=0) C C$ \\
\hline DPF-32B & $\mathrm{CCC}(=0) \mathrm{O} / \mathrm{N}=\mathrm{C}(/ \mathrm{C}) \mathrm{C}(=0) \mathrm{c}(\mathrm{c} 1) \mathrm{ccc}(\mathrm{c} 12) \mathrm{c} 3 \mathrm{c}(\mathrm{C} 2(\mathrm{CCC}) \mathrm{CCC}) \mathrm{cccc} 3$ \\
\hline DPS-31B & $\mathrm{CC}(=0) \mathrm{O} / \mathrm{N}=\mathrm{C}(\backslash \mathrm{C}) \mathrm{C}(=0) \mathrm{c} 1 \mathrm{ccc}(\mathrm{cc} 1) \mathrm{Sc} 2 \mathrm{ccccc} 2$ \\
\hline FA-31A & $\mathrm{CC}(=0) \mathrm{O} / \mathrm{N}=\mathrm{C}(\backslash \mathrm{CC}) \mathrm{c}(\mathrm{cc} 1) \mathrm{cc}(\mathrm{c} 12) \mathrm{c} 3 \mathrm{c} 4 \mathrm{c} 2 \mathrm{cccc} 4 \mathrm{ccc} 3$ \\
\hline FA-81A & $\mathrm{c} 1 \mathrm{ccc} 2 \operatorname{cccc} 3 c(\operatorname{coc} 1 \mathrm{c} 23) \operatorname{ccc}(\mathrm{c} 4) / \mathrm{C}(=\mathrm{N} \backslash \mathrm{OC}(=0) \mathrm{C}) \mathrm{ccccccc}$ \\
\hline FA-81B & $\operatorname{ccccccC}(=N \backslash O C(=0) C) \backslash C(=0) c(c c 1) \operatorname{cc}(c 12) c 3 c 4 c 2 \operatorname{cccc} 4 \operatorname{ccc} 3$ \\
\hline FA-86A & $\operatorname{c1ccc}(\operatorname{coc} 13) \operatorname{c} 4 \mathrm{c}(\mathrm{c} 2 \mathrm{ccc} 3) \mathrm{cc}(\mathrm{cc} 4) / \mathrm{C}(\mathrm{CcccccC})=\mathrm{N} / \mathrm{OC}(=0) \mathrm{cccccC}$ \\
\hline FL-N19 & $\mathrm{CC}(=0) \mathrm{O} / \mathrm{N}=\mathrm{C}(\backslash \mathrm{C}) \mathrm{C}(=0) \mathrm{c}(\operatorname{ccc} 1) \mathrm{c}(\mathrm{c} 12) \operatorname{cccc} 2$ \\
\hline FL-N21 & $\mathrm{CC}(=0) \mathrm{O} / \mathrm{N}=\mathrm{C}(\backslash \mathrm{C}) \mathrm{C}(=0) \mathrm{c}(\mathrm{c} 1) \mathrm{ccc}(\mathrm{c} 12) \mathrm{c} 3 \mathrm{c}(\mathrm{C} 2(\mathrm{CC}) \mathrm{CC}) \mathrm{cc}(\mathrm{Br}) \mathrm{cc} 3$ \\
\hline FL-N26 & $\operatorname{c1cccc}(\operatorname{c12}) \mathrm{C}(\mathrm{CCCC})(\mathrm{CCCC}) \mathrm{c} 3 \mathrm{c} 2 \mathrm{ccc}(\mathrm{c} 3) \mathrm{C}(=0) / \mathrm{C}(=\mathrm{N} \backslash \mathrm{OC}(=0) \mathrm{C}) \mathrm{C}(=0) \mathrm{c} 4 \mathrm{ccccc} 4$ \\
\hline FL-N30 & $\mathrm{CC}(=0) \mathrm{O} / \mathrm{N}=\mathrm{C}(\backslash \mathrm{C}) \mathrm{C}(=0) \mathrm{c}(\mathrm{c} 1) \mathrm{ccc}(\mathrm{c} 12) \mathrm{c} 3 \mathrm{c}(\mathrm{C} 2(\mathrm{CCCC}) \mathrm{CCCC}) \mathrm{cc}(\mathrm{cc} 3)-\mathrm{c} 4 \mathrm{onc}(\mathrm{c} 4) \mathrm{C}$ \\
\hline FL-N38 & $\mathrm{CC}(=0) \mathrm{O} / \mathrm{N}=\mathrm{C}(/ \mathrm{C}) \mathrm{C}(=\mathrm{O}) \mathrm{c}(\mathrm{c} 1) \mathrm{ccc}(\mathrm{c} 12) \mathrm{c} 3 \mathrm{c}([\mathrm{C} @ \mathrm{H}] 2 \mathrm{CC}) \mathrm{cccc} 3$ \\
\hline FL-N40 & $C C(=0) 0 / N=C(/ C) C(=0) c(c 1) c c c(c 12) c 3 c(C 2(C) C) c c(c c 3) C(=0) / C(C)=N / O C(=0) C$ \\
\hline FL-N41 & $c 1 \operatorname{ccccc} 1 C(=0) c(\operatorname{cc} 2) \operatorname{cc}(c 23) c(C C C C)(C C C C) c 4 c 3 c c c(c 4) / C(C)=N / O C(=0) C$ \\
\hline FL-N42 & $\operatorname{c1cccc}(\mathrm{c} 12) \operatorname{ccc}(\mathrm{c} 2) \mathrm{C}(=0) \mathrm{c}(\mathrm{cc} 3) \mathrm{cc}(\mathrm{c} 34) \mathrm{C}(\mathrm{CCCC})(\mathrm{CCCC}) \mathrm{c} 5 \mathrm{c} 4 \mathrm{ccc}(\mathrm{c} 5) / \mathrm{C}(\mathrm{C})=\mathrm{N} / \mathrm{OC}(=0) \mathrm{C}$ \\
\hline FL-N49 & $\operatorname{c1cccc}(\operatorname{c12}) \mathrm{C}(\mathrm{CCCC})(\mathrm{CCCC}) \mathrm{c} 3 \mathrm{c} 2 \mathrm{ccc}(\mathrm{c} 3) \mathrm{C}(=\mathrm{O}) / \mathrm{C}(=\mathrm{N} \backslash \mathrm{OC}(=0) \mathrm{C}) \mathrm{c} 4 \mathrm{ccccc} 4$ \\
\hline FL-N52 & $C C(=0) 0 / N=C(\backslash C) C(=0) c 1 c c c(c c 1)-c(\operatorname{ccc} 2) c(c 23) \operatorname{cccc} 3$ \\
\hline FL-N54 & $\mathrm{O}=[\mathrm{N}+]([\mathrm{O}-]) \mathrm{c} 1 \mathrm{ccc}(\mathrm{cc} 1) / \mathrm{C}(=\mathrm{N} \backslash \mathrm{OC}(=\mathrm{O}) \mathrm{C}) \mathrm{c}(\mathrm{c} 2) \operatorname{ccc}(\mathrm{c} 23) \mathrm{c} 4 \mathrm{c}(\mathrm{cc}([\mathrm{N}+](=\mathrm{O})[\mathrm{O}-]) \mathrm{cc} 4) \mathrm{C} 3(\mathrm{CCCC}) \mathrm{CCCC}$ \\
\hline FL-N55 & $\operatorname{c1cc}([N+]([0-])=0) \operatorname{cc}(\mathrm{c} 12) \mathrm{c}(\mathrm{CccC})(\mathrm{CcCC}) \operatorname{coc} 2 \operatorname{ccc}(\mathrm{c} 3) / \mathrm{C}(\mathrm{c} 4 \mathrm{ccc}([\mathrm{N}+](=0)[0-]) \operatorname{cc} 4)=\mathrm{N} / \mathrm{OC}(=0) \operatorname{c5} \operatorname{ccccc} 5$ \\
\hline FL-N56 & $C C(=0) O / N=C(\backslash C) C(=0) \operatorname{coccc}(\operatorname{cc} 1) \operatorname{Sc} 2 \operatorname{ccc}(\operatorname{cc} 2) C(=0) / C(C)=N / O C(=0) C$ \\
\hline FL-N60 & $\mathrm{CC}(=0) \mathrm{O} / \mathrm{N}=\mathrm{C}(/ \mathrm{C}) \mathrm{C}(=0) \mathrm{c} 1 \mathrm{ccc}(\mathrm{cc} 1)-\mathrm{c} 2 \mathrm{ccc}(\mathrm{cc} 2) \mathrm{OC}$ \\
\hline FL-N68 & $\mathrm{CC}(=0) \mathrm{O} / \mathrm{N}=\mathrm{C}(/ \mathrm{C}) \mathrm{C}(=0) \mathrm{c} 1 \mathrm{ccc}(\mathrm{cc} 1)-\mathrm{c} 2 \mathrm{ccc}(\mathrm{cc} 2) \operatorname{Sc} 3 \operatorname{ccccc} 3$ \\
\hline FL-N88 & $\mathrm{c} 1 \mathrm{cc}(\mathrm{OC}) \mathrm{cc}(\mathrm{c} 12) \mathrm{C}(\mathrm{CC})(\mathrm{CC}) \mathrm{c} 3 \mathrm{c} 2 \mathrm{ccc}(\mathrm{c} 3) \mathrm{C}(=\mathrm{O}) / \mathrm{C}(\mathrm{C})=\mathrm{N} / \mathrm{OC}(=0) \mathrm{C}$ \\
\hline IB-261 & $\operatorname{cccccc} / \mathrm{C}(=\mathrm{N} \backslash \mathrm{OC}(=0) \mathrm{C}) \mathrm{c}(\mathrm{cn} 1 \mathrm{CC}) \mathrm{c}(\mathrm{c} 12) \mathrm{cc}([\mathrm{N}+]([\mathrm{O}-])=0) \mathrm{cc} 2$ \\
\hline MP-N104 & $\mathrm{CC}(=0) \mathrm{O} / \mathrm{N}=\mathrm{C}(\backslash \mathrm{C}) \mathrm{C}(=0) \mathrm{c} 1 \mathrm{ccc}(\mathrm{cc} 1) \mathrm{N} 2 \mathrm{CCOCC} 2$ \\
\hline N1919 & $\mathrm{O}=\mathrm{C}(\mathrm{C}) \mathrm{O} \backslash \mathrm{N}=\mathrm{C}(\mathrm{C}) \backslash \mathrm{c}(\mathrm{c} 1) \mathrm{ccc}(\mathrm{c} 12) \mathrm{n}(\mathrm{CC}) \mathrm{c} 3 \mathrm{c} 2 \mathrm{cc}(\mathrm{cc} 3) \mathrm{C}(=\mathrm{O}) \mathrm{c4c}(\mathrm{C}) \mathrm{cc}(\mathrm{cc} 4) \mathrm{O}[\mathrm{C} @ @ \mathrm{H}](\mathrm{CO}) \mathrm{OC5}(\mathrm{C}) \mathrm{C}$ \\
\hline NBF-61A & $c 1 c c([N+]([0-])=0) c c(c 12) C(C C C C)(C C C C) c 3 c 2 c c c(c 3) / C(=N / O C(=0) C) c c c c C$ \\
\hline NBF-CyA1 & $\operatorname{c1cc}([\mathrm{N}+]([\mathrm{O}-])=0) \operatorname{cc}(\mathrm{c} 12) \mathrm{C}(\mathrm{CCCC})(\mathrm{CccC}) \mathrm{c} 3 \mathrm{c} 2 \mathrm{ccc}(\mathrm{c} 3) / \mathrm{C}(=\mathrm{N} / \mathrm{OC}(=\mathrm{O}) \mathrm{C}) \mathrm{C} 4 \mathrm{Ccccc} 4$ \\
\hline
\end{tabular}




\begin{tabular}{|c|c|}
\hline NBP-31A & $\operatorname{c1cc}([\mathrm{N}+](=0)[0-]) \operatorname{ccc} 1-\mathrm{c} 2 \mathrm{ccc}(\mathrm{cc} 2) / \mathrm{c}(\mathrm{CC})=\mathrm{N} / \mathrm{OC}(=\mathrm{O}) \mathrm{C}$ \\
\hline NBP-51A & $\operatorname{c1cc}([\mathrm{N}+](=0)[0-]) \operatorname{ccc} 1-\operatorname{coccc}(\operatorname{cc} 2) / \mathrm{C}(=\mathrm{N} / \mathrm{OC}(=0) \mathrm{c}) \mathrm{cccc}$ \\
\hline NBP-71A & $\operatorname{c1cc}([\mathrm{N}+](=0)[0-]) \operatorname{ccc} 1-\operatorname{coccc}(\operatorname{cc} 2) / \mathrm{C}(=\mathrm{N} / \mathrm{OC}(=0) \mathrm{C}) \operatorname{cccccC}$ \\
\hline NDPF-41A & $\mathrm{CC}(=0) \mathrm{O} / \mathrm{N}=\mathrm{C}(\backslash \mathrm{CCC}) \mathrm{c}(\mathrm{c} 1) \mathrm{ccc}(\mathrm{c} 12) \mathrm{c} 3 \mathrm{c}(\mathrm{C} 2(\mathrm{CCC}) \mathrm{CCC}) \mathrm{cc}([\mathrm{N}+](=0)[0-]) \mathrm{cc} 3$ \\
\hline OXE-01 & $\operatorname{c1} 1 \operatorname{ccccc} 1 \mathrm{C}(=0) \mathrm{O} / \mathrm{N}=\mathrm{C}(\mathrm{CCCCCCC}) / \mathrm{C}(=0) \operatorname{coccc}(\operatorname{cc} 2) \operatorname{Sc} 3 \operatorname{ccccc} 3$ \\
\hline OXE-02 & $\mathrm{CC}(=0) \mathrm{O} / \mathrm{N}=\mathrm{C}(/ \mathrm{C}) \mathrm{c}(\mathrm{cc} 1) \mathrm{cc}(\mathrm{c} 12) \mathrm{c} 3 \mathrm{c}(\mathrm{n} 2 \mathrm{CC}) \mathrm{ccc}(\mathrm{c} 3) \mathrm{C}(=0) \mathrm{c} 4 \mathrm{c}(\mathrm{C}) \mathrm{cccc} 4$ \\
\hline OXE-03 & $\mathrm{FC}(\mathrm{F}) \mathrm{C}(\mathrm{F})(\mathrm{F}) \mathrm{C}(\mathrm{F})(\mathrm{F}) \mathrm{C}(\mathrm{F})(\mathrm{F}) 0 \mathrm{c} 1 \mathrm{ccccc} 1 / \mathrm{C}(=\mathrm{N} / \mathrm{OC}(=0) \mathrm{C}) \mathrm{c}(\mathrm{cc} 2) \mathrm{cc}(\mathrm{c} 23) \mathrm{c} 4 \mathrm{c}(\mathrm{n} 3 \mathrm{C}[\mathrm{C} @ \mathrm{H}](\mathrm{CC}) \mathrm{CCCC}) \mathrm{c} 5 \mathrm{c}(\mathrm{cccc} 5) \mathrm{c}(\mathrm{c} 4) \mathrm{C}(=0) \mathrm{c} 6 \mathrm{c}(\mathrm{C}) \mathrm{cc}(\mathrm{C}) \mathrm{cc} 6 \mathrm{C}$ \\
\hline PBG-304 & $\mathrm{C} 1 \mathrm{CCCC} 1 \mathrm{CC} / \mathrm{C}(=\mathrm{N} \backslash \mathrm{OC}(=0) \mathrm{C}) \mathrm{c}(\mathrm{cc} 2) \mathrm{cc}(\mathrm{c} 23) \mathrm{c} 4 \mathrm{c}(\mathrm{n} 3 \mathrm{CC}) \mathrm{ccc}(\mathrm{c} 4) \mathrm{C}(=0) \mathrm{c5} \mathrm{c}(\mathrm{C}) \mathrm{cccc} 5$ \\
\hline POE & $\mathrm{c} 12 \mathrm{c} 3 \mathrm{c} 4 \mathrm{ccc} 1 \mathrm{c}(/ \mathrm{C}(\mathrm{C})=\mathrm{N} / \mathrm{OC}(=\mathrm{O}) \mathrm{C}) \operatorname{ccc} 2 \mathrm{ccc} 3 \mathrm{ccc} 4$ \\
\hline PTAL-21B & $C C(=0) 0 / N=C(\backslash C) C(=0) c(c 1) c c c(c 12) N(C(=0) C) c 3 c(S 2) \operatorname{cccc} 3$ \\
\hline PTB-21B & $\mathrm{CC}(=0) \mathrm{O} / \mathrm{N}=\mathrm{C}(\backslash \mathrm{C}) \mathrm{C}(=0) \mathrm{c}(\mathrm{c} 1) \mathrm{ccc}(\mathrm{c} 12) \mathrm{N}(\mathrm{CCCC}) \mathrm{c} 3 \mathrm{c}(\mathrm{S} 2) \mathrm{cccc} 3$ \\
\hline PTE-21B & $\mathrm{CC}(=0) \mathrm{O} / \mathrm{N}=\mathrm{C}(\backslash \mathrm{C}) \mathrm{C}(=0) \mathrm{c}(\mathrm{c} 1) \mathrm{ccc}(\mathrm{c} 12) \mathrm{N}(\mathrm{CC}) \mathrm{c} 3 \mathrm{c}(\mathrm{S} 2) \operatorname{cccc} 3$ \\
\hline QBB-31 & $\mathrm{CC}(=0) 0 / \mathrm{N}=\mathrm{C}(\backslash \mathrm{C}) \mathrm{C}(=0) \mathrm{c}(\mathrm{c}(\mathrm{c} 12) \operatorname{ccc} 2) \operatorname{ccc} 10 \mathrm{Cc} 3 \mathrm{ccccc} 3$ \\
\hline SBC-31B & $C C(=0) O / N=C(\backslash C) C(=0) c 1 c(0) c(c c(C) c 1) C(=0) / C(C)=N / O C(=0) C$ \\
\hline SBF-31B & $\mathrm{O}=\mathrm{C}(\mathrm{C}) \mathrm{O} \backslash \mathrm{N}=\mathrm{C}(\mathrm{C}) \backslash \mathrm{C}(=\mathrm{O}) \mathrm{c}(\mathrm{cc} 1) \mathrm{cc}(\mathrm{c} 12) \mathrm{C}(\mathrm{CCCC})(\mathrm{CCCC}) \mathrm{c} 3 \mathrm{c} 2 \mathrm{ccc}(\mathrm{c} 3) \mathrm{C}(=0) / \mathrm{C}(\mathrm{C})=\mathrm{N} / \mathrm{OC}(=0) \mathrm{C}$ \\
\hline SBF-41B & $\mathrm{O}=\mathrm{C}(\mathrm{C}) \mathrm{O} \backslash \mathrm{N}=\mathrm{C}(\mathrm{CC}) \backslash \mathrm{C}(=\mathrm{O}) \mathrm{c}(\mathrm{cc} 1) \mathrm{cc}(\mathrm{c} 12) \mathrm{C}(\mathrm{CCCC})(\mathrm{CCCC}) \mathrm{c} 3 \mathrm{c} 2 \mathrm{ccc}(\mathrm{c} 3) \mathrm{C}(=0) / \mathrm{C}(\mathrm{CC})=\mathrm{N} / \mathrm{OC}(=0) \mathrm{C}$ \\
\hline SBF-71A & $\operatorname{ccccccC}(=N / O C(=0) C) \backslash c(c c 1) c c(c 12) C(C C C C)(C C C C) c 3 c 2 c c c(c 3) / C(=N / O C(=0) C) C C C C C C$ \\
\hline SBF-71B-E & $\operatorname{ccccccC}(=N \backslash O C(=0) C) \backslash C(=0) c(\operatorname{cc} 1) \operatorname{cc}(c 12) C(C C C C)(C C C C) c 3 c 2 c c c(c 3) C(=0) / C(=N \backslash O C(=0) C) C C C C C$ \\
\hline SBF-71B-Z & $\operatorname{cccccC}(=N / O C(=0) C) \backslash C(=0) c(\operatorname{cc} 1) \operatorname{cc}(\operatorname{c1} 12) C(C C C C)(C C C C) c 3 c 2 \operatorname{ccc}(c 3) C(=0) / C(=N / O C(=0) C) \operatorname{ccccC}$ \\
\hline SBP-31B & $C C(=0) O / N=C(/ C) C(=0) c 1 c c c(\operatorname{cc} 1)-c 2 c c c(\operatorname{cc} 2) C(=0) / C(C)=N \backslash O C(=0) C$ \\
\hline SBP-71B & $\operatorname{cccccC}(=N \backslash O C(=0) C) \backslash C(=0) c(\operatorname{cc} 1) \operatorname{ccc} 1-c 2 \operatorname{ccc}(\operatorname{cc} 2) C(=0) / C(=N / O C(=0) C) C C C C C$ \\
\hline SEF-31B & $C C(=0) O \backslash N=C(C) \backslash C(=0) c(c c 1) c c(c 12) C(C C)(C C) c 3 c 2 c c c(c 3) C(=0) / C(C)=N / O C(=0) C$ \\
\hline SEF-41B & $C C(=0) O / N=C(\backslash C C) C(=0) c(c 1) c c c(c 12) c 3 c(C 2(C C) C C) c c(c c 3) C(=0) / C(C C)=N / O C(=0) C$ \\
\hline SEF-51B & $C C(=0) O / N=C(\backslash C C C) C(=0) c(c 1) \operatorname{ccc}(c 12) c 3 c(C 2(C C) C C) c c(c c 3) C(=0) / C(C C C)=N / O C(=0) C$ \\
\hline SEF-3031B & $C C C(=0) c(c c 1) c c(c 12) C(C C)(C C) c 3 c 2 c c c(c 3) C(=0) / C(C)=N / O C(=0) C$ \\
\hline SEF-P21B & $\operatorname{c1cccc} 1 \mathrm{C}(=\mathrm{N} / \mathrm{OC}(=0) \mathrm{C}) \backslash \mathrm{C}(=0) \mathrm{c}(\mathrm{cc} 2) \operatorname{cc}(\mathrm{c} 23) \mathrm{C}(\mathrm{CC})(\mathrm{CC}) \mathrm{c} 4 \mathrm{c} 3 \mathrm{ccc}(\mathrm{c} 4) \mathrm{C}(=\mathrm{O}) / \mathrm{C}(=\mathrm{N} / \mathrm{OC}(=0) \mathrm{C}) \mathrm{c} 5 \operatorname{ccccc} 5$ \\
\hline SPF-41B & $C C(=0) O / N=C(\backslash C C) C(=0) c(c 1) c c c(c 12) C 3=C(C 2(C C C) C C C) C[C @ H](C=C 3) C(=0) / C(C C)=N / O C(=0) C$ \\
\hline SPF-51B & $C C(=0) O / N=C(\backslash C C C) C(=0) c(c 1) \operatorname{ccc}(c 12) c 3 c(C 2(C C C) C C C) c c(c c 3) C(=0) / C(C C C)=N / O C(=0) C$ \\
\hline SPI-01 & $\operatorname{c1cc}([\mathrm{N}+]=0)[0-]) \operatorname{ccc} 1-c 2 c c c(\operatorname{cc} 2) \mathrm{C}(=0) / c(c c c c C)=\mathrm{N} / O C(=0) c c$ \\
\hline
\end{tabular}




\begin{tabular}{|c|c|}
\hline SPI-02 & $\mathrm{CC}(=0) \mathrm{O} / \mathrm{N}=\mathrm{C}(\backslash \mathrm{C}) \mathrm{c}(\mathrm{c} 1) \operatorname{ccc}(\mathrm{c} 12) \mathrm{c} 3 \mathrm{c}(\mathrm{cc}([\mathrm{N}+]([\mathrm{O}-])=0) \operatorname{cc} 3) \mathrm{C2}(\mathrm{cccC}) \mathrm{cccc}$ \\
\hline SPI-03 & $\mathrm{CC}(=0) 0 / \mathrm{N}=\mathrm{C}(/ \mathrm{C}) \mathrm{C}(=0) \mathrm{c}(\mathrm{c} 1) \mathrm{ccc}(\mathrm{c} 12) \mathrm{c} 3 \mathrm{c}(\mathrm{C2}(\mathrm{CC}) \mathrm{CC}) \operatorname{cccc} 3$ \\
\hline SPI-03E & $C C C(=0) 0 / N=C(\backslash C) C(=0) c(c 1) c c c(c 12) c 3 c(C 2(C C) C C) c c c c 3$ \\
\hline SPTD-41B & $C C(=0) 0 / N=C(\backslash C C) C(=0) c(c 1) \operatorname{ccc}(c 12) N(c 3 c(S 2) c c(c c 3) C(=0) / C(C C)=N / O C(=0) C) c c c c c c c c c$ \\
\hline SPTAC-41B & $C C(=0) O \backslash N=C(C C) \backslash C(=0) c(c c 1) c c(c 12) S c 3 c(N 2 C(=0) C) c c c(c 3) C(=0) / C(C C)=N / O C(=0) C$ \\
\hline SPTB-41B & $C C(=0) O / N=C(\backslash C C) C(=0) c(c 1) \operatorname{ccc}(c 12) N(C C C C) c 3 c(S 2) c c(c c 3) C(=0) / C(C C)=N / O C(=0) C$ \\
\hline SPTE-21A & $\mathrm{CC}(=0) \mathrm{O} / \mathrm{N}=\mathrm{C}(\backslash \mathrm{CC}) \mathrm{c}(\mathrm{c} 1) \mathrm{ccc}(\mathrm{c} 12) \mathrm{N}(\mathrm{CC}) \mathrm{c} 3 \mathrm{c}(\mathrm{S} 2) \mathrm{cc}(\mathrm{cc} 3) / \mathrm{C}(\mathrm{CC})=\mathrm{N} / \mathrm{OC}(=\mathrm{O}) \mathrm{C}$ \\
\hline SPTEH-41B & $C C(=0) O / N=C(\backslash C C) C(=0) c(c 1) \operatorname{ccc}(c 12) N(C[C @ H](C C) C C C C) c 3 c(S 2) c c(c c 3) C(=0) / C(C C)=N / O C(=0) C$ \\
\hline SPTHx-41B & $O=C(C) O \backslash N=C(C C) \backslash C(=0) c(c c 1) c c(c 12) \operatorname{Sc} 3 c(N 2 C C C C C C) c c c(c 3) C(=0) / C(C C)=N \backslash O C(=0) C$ \\
\hline SPTO-41B & $O=C(C) O \backslash N=C(C C) \backslash C(=0) c(c c 1) c c(c 12) S c 3 c(N 2 C c c c c c c C) c c c(c 3) C(=0) / C(C C)=N \backslash O C(=0) C$ \\
\hline SPTO-71B & $\operatorname{cccccc}(=\mathrm{N} / O C(=0) C) \backslash C(=0) c(\operatorname{cc} 1) \operatorname{cc}(\mathrm{c} 12) \operatorname{Sc} 3 c(\mathrm{~N} 2 \operatorname{ccccccc} C) \operatorname{ccc}(\mathrm{c} 3) \mathrm{C}(=0) / \mathrm{C}(=\mathrm{N} / \mathrm{OC}(=0) \mathrm{C}) \operatorname{cccc}$ \\
\hline
\end{tabular}

\section{Details of experimental setups}

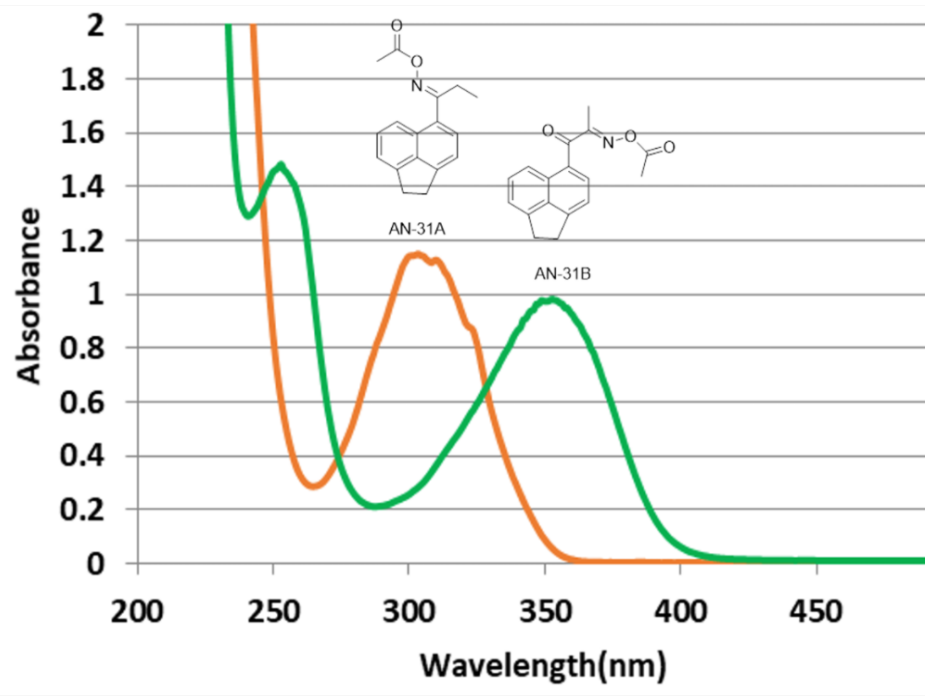

Figure S1. Absorption spectra of representative oxime ester (AN-31A, orange) and keto-oxime ester (AN31B, green) photoinitiator candidates.

\subsection{Preparation of binder resin}

After putting $200 \mathrm{ml}$ PGMEA and $1.5 \mathrm{~g}$ AIBN into a $500 \mathrm{ml}$ polymerization container, acrylic monomers were added at a mole ratio of 20:20:40:20 of methacrylic acid, glycidyl methacrylate, methyl methacrylate and dicyclopentenyl acrylate, respectively, with $40 \mathrm{wt} \%$ solids, followed by polymerization while stirring under nitrogen atmosphere at $70^{\circ} \mathrm{C}$ for 5 hours to prepare acrylic polymer, i.e., binder resin 1 . The average molecular weight of the resulting copolymer was measured at 25,000, and the degree of dispersion at 2.0. 


\subsection{Preparation of photoresist compositions}

Photoresist compositions were prepared by putting binder resins, photoinitiators, and $0.1 \mathrm{wt} \% \mathrm{FC}-430$ (3M leveling agent) into a reaction mixing tank with a UV shield and a stirrer in a sequential order according to the ingredients. After stirring at room temperature, the balance of PGMEA as a solvent was added such that the total $w t \%$ of the composition is $100 \mathrm{wt} \%$.

\subsection{Evaluation of photoresist compositions with photosensitivity}

Each photoresist prepared from the same binder resin and the different photoinitiator was spin-coated onto the glass substrate and dried at $100^{\circ} \mathrm{C}$ on a hotplate for 2 minutes. It was then exposed to light using a step mask and development in $0.045 \% \mathrm{KOH}$ aqueous solution. The sensitivity was evaluated based on an exposure dose at which the thickness of a step mask pattern was maintained at $80 \%$ of an initial thickness.

\subsection{Photoreaction mechanisms}

Figure S2 illustrates the mechanisms of the photoreaction by the types of photoinitiators examined in this work.

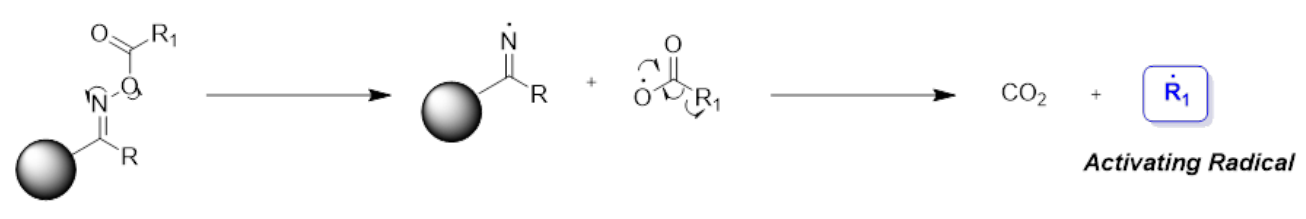

(a)

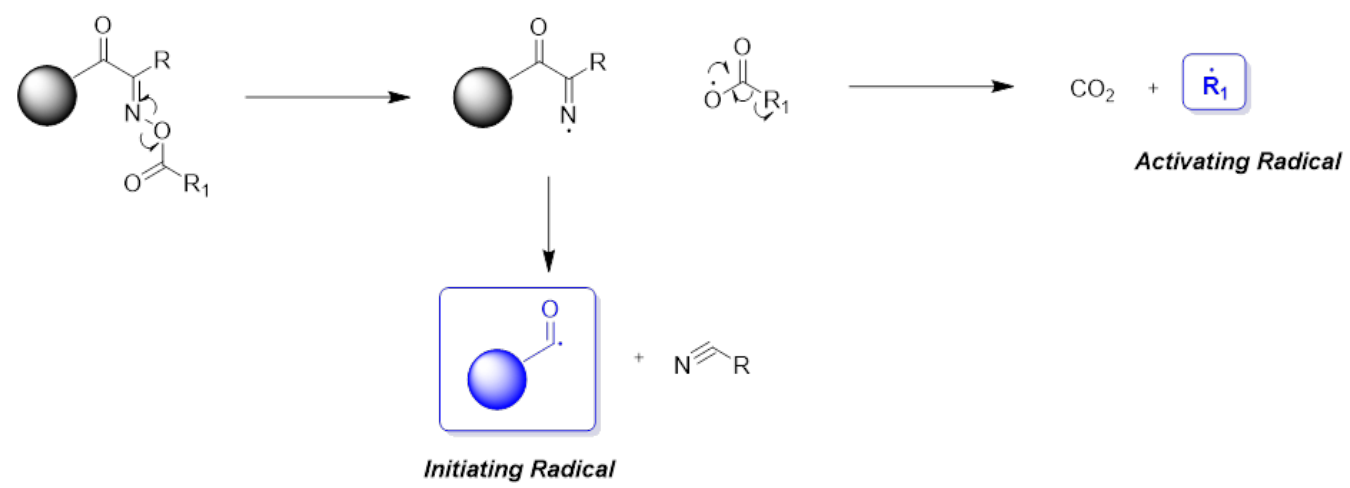

(b)

Figure S2. Illustrations of photoreaction mechanisms for (a) oxime ester type and (b) keto-oxime ester type photoinitiators synthesized and examined in this work. 


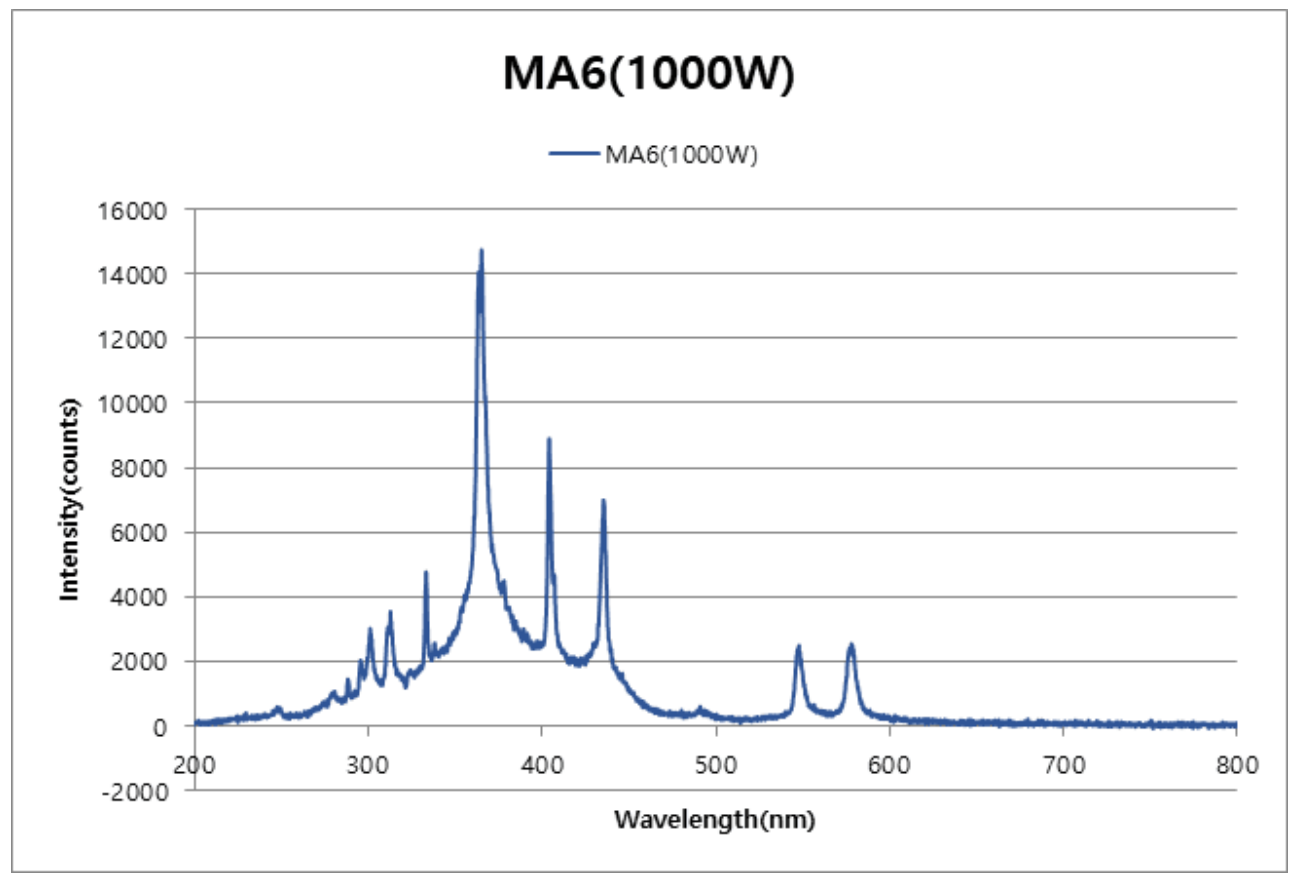

(a)

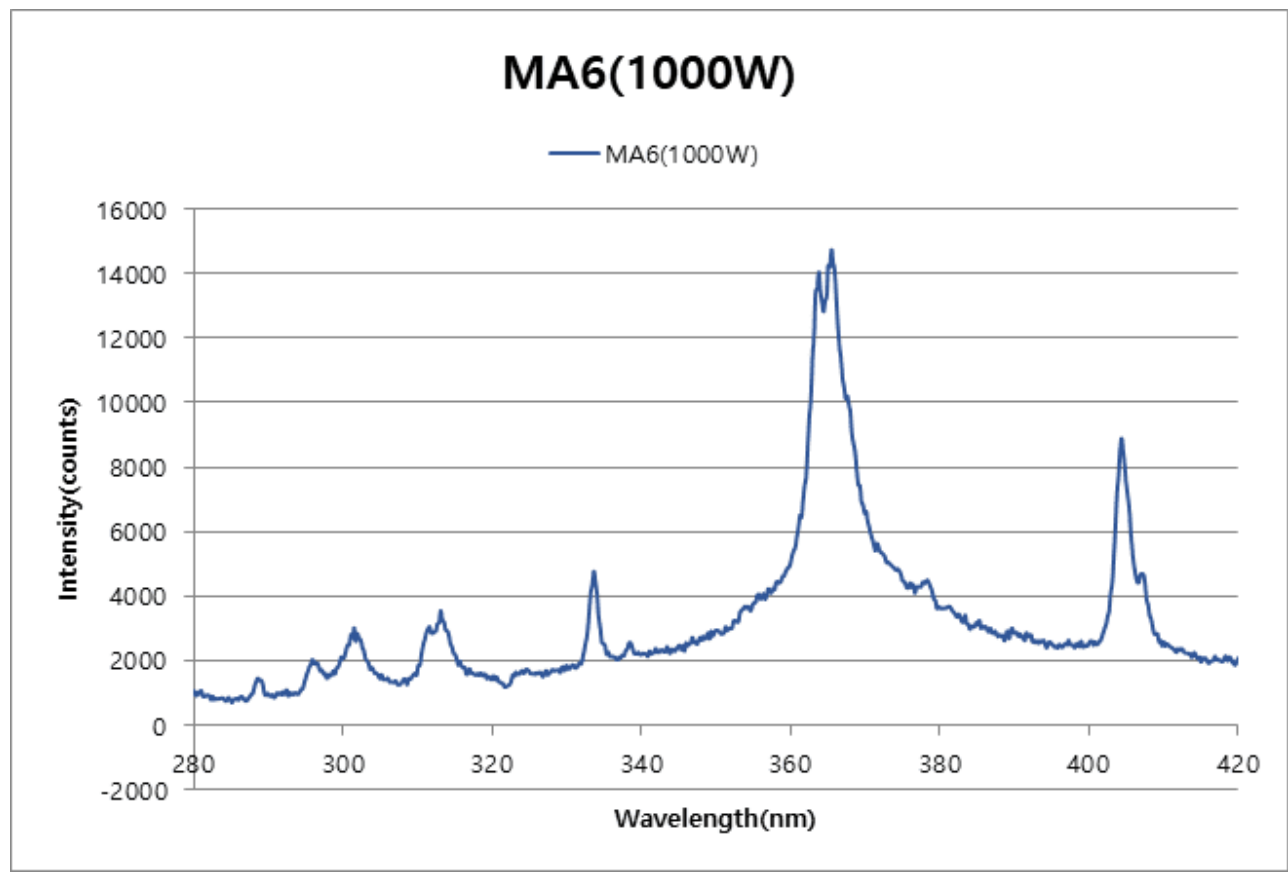

(b)

Figure S3. Emission spectrum from the mask aligner plotted between $200 \mathrm{~nm}$ and $800 \mathrm{~nm}$ (a) and between $280 \mathrm{~nm}$ and $420 \mathrm{~nm}$ (b). 


\section{Summary of performance data for machine learning models}
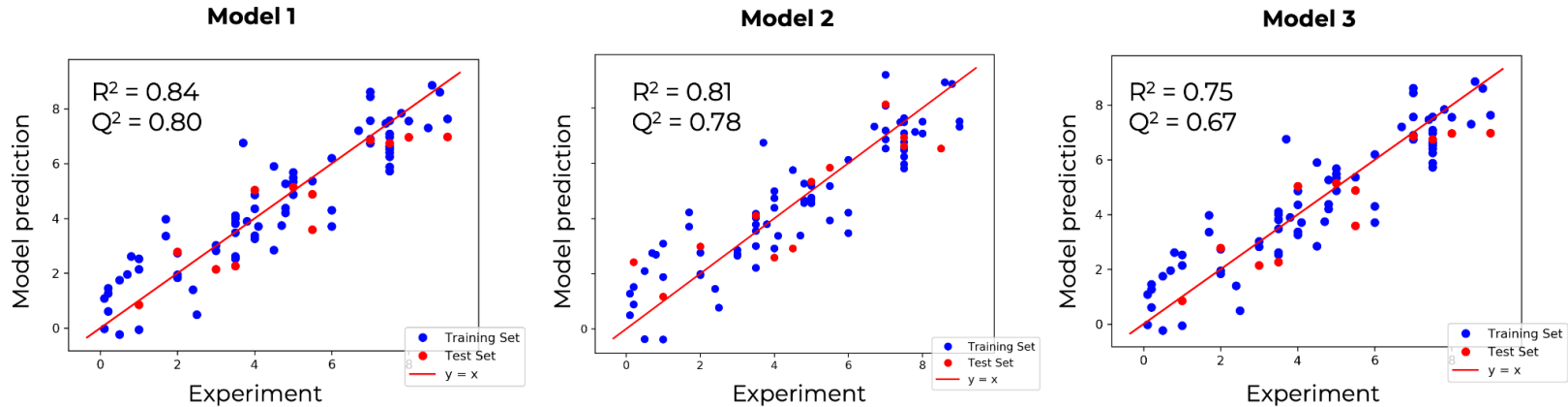

Figure S4. Scatter plots from the three best-performing continuous models for photosensitivity prediction.

Table S2. Confusion matrix elements from the top-five categorical models to predict zero photosensitivity from photoinitiator candidates.

\begin{tabular}{|c|c|c|c|c|c|c|}
\hline \multicolumn{7}{|c|}{ TRAINING SET } \\
\hline Model & Type & Score & $\begin{array}{l}\text { Predicted zero \& } \\
\text { Measured zero }\end{array}$ & $\begin{array}{c}\text { Predicted non-zero \& } \\
\text { Measured non-zero }\end{array}$ & $\begin{array}{c}\text { Predicted zero \& } \\
\text { Measured non-zero }\end{array}$ & $\begin{array}{l}\text { Predicted non-zero \& } \\
\text { Measured zero }\end{array}$ \\
\hline 1 & Recursive Partitioning & 0.8805 & 29 & 5 & 7 & 64 \\
\hline 2 & Recursive Partitioning & 0.8800 & 28 & 6 & 5 & 66 \\
\hline 3 & Recursive Partitioning & 0.8736 & 28 & 6 & 5 & 66 \\
\hline 4 & Recursive Partitioning & 0.8702 & 28 & 6 & 6 & 65 \\
\hline \multirow[t]{3}{*}{5} & Recursive Partitioning & 0.8640 & 28 & 6 & 7 & 64 \\
\hline & & SUM & 141 & 29 & 30 & 325 \\
\hline & & $\%$ & 26.86 & 5.52 & 5.71 & 61.90 \\
\hline \multicolumn{7}{|c|}{ TEST SET } \\
\hline Model & Type & Score & $\begin{array}{l}\text { Predicted zero \& } \\
\text { Measured zero }\end{array}$ & $\begin{array}{l}\text { Predicted non-zero \& } \\
\text { Measured non-zero }\end{array}$ & $\begin{array}{c}\text { Predicted zero \& } \\
\text { Measured non-zero }\end{array}$ & $\begin{array}{c}\text { Predicted non-zero \& } \\
\text { Measured zero }\end{array}$ \\
\hline 1 & Recursive Partitioning & 0.8805 & 6 & 0 & 1 & 11 \\
\hline 2 & Recursive Partitioning & 0.8800 & 6 & 0 & 1 & 11 \\
\hline 3 & Recursive Partitioning & 0.8736 & 5 & 1 & 1 & 11 \\
\hline 4 & Recursive Partitioning & 0.8702 & 5 & 1 & 1 & 11 \\
\hline \multirow[t]{3}{*}{5} & Recursive Partitioning & 0.8640 & 5 & 1 & 1 & 11 \\
\hline & & SUM & 27 & 3 & 5 & 55 \\
\hline & & $\%$ & 30.00 & 3.33 & 5.56 & 61.11 \\
\hline \multicolumn{7}{|l|}{$\overline{\text { ALL }}$} \\
\hline Model & Type & Score & $\begin{array}{l}\text { Predicted zero \& } \\
\text { Measured zero }\end{array}$ & $\begin{array}{l}\text { Predicted non-zero \& } \\
\text { Measured non-zero }\end{array}$ & $\begin{array}{c}\text { Predicted zero \& } \\
\text { Measured non-zero }\end{array}$ & $\begin{array}{l}\text { Predicted non-zero \& } \\
\text { Measured zero }\end{array}$ \\
\hline 1 & Recursive Partitioning & 0.8805 & 35 & 5 & 8 & 75 \\
\hline 2 & Recursive Partitioning & 0.8800 & 34 & 6 & 6 & 77 \\
\hline 3 & Recursive Partitioning & 0.8736 & 33 & 7 & 6 & 77 \\
\hline 4 & Recursive Partitioning & 0.8702 & 33 & 7 & 7 & 76 \\
\hline \multirow[t]{3}{*}{5} & Recursive Partitioning & 0.8640 & 33 & 7 & 8 & 75 \\
\hline & & SUM & 168 & 32 & 35 & 380 \\
\hline & & $\%$ & 27.32 & 5.20 & 5.69 & 61.79 \\
\hline
\end{tabular}

\section{Additional model visualization examples from the best-performing continuous model}



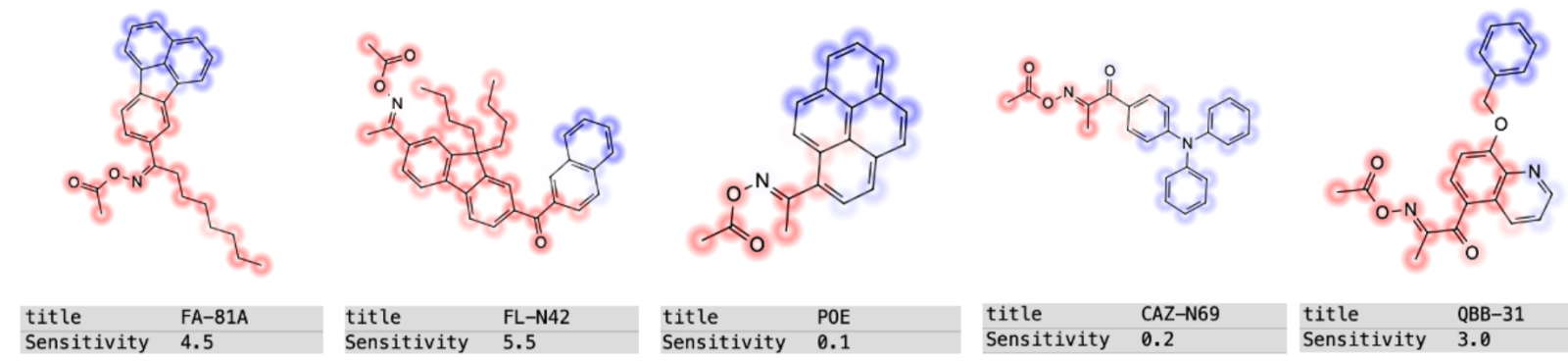

Sensitivity 0.1

titl

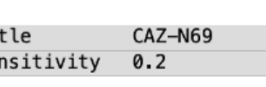

title QBB-31

Figure S5. Model visualization from the best-performing continuous model for photosensitivity prediction showing the predictive trend of fused rings (FA-81A, FL-N42, POE) or free-rotating phenyl groups (CAZN69, QBB-31) decreasing the photosensitivity.

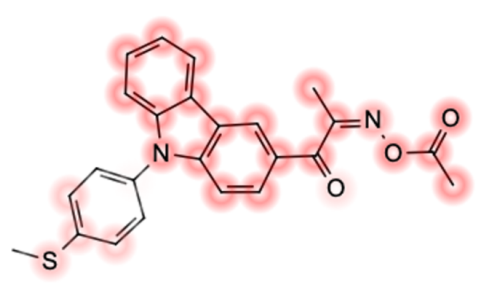

1

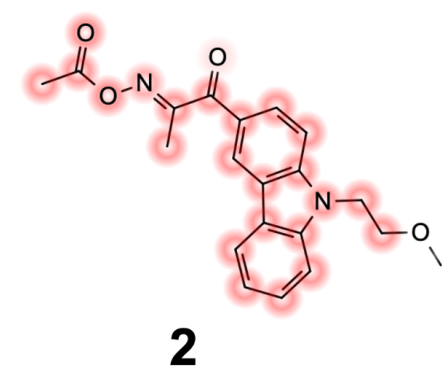

2

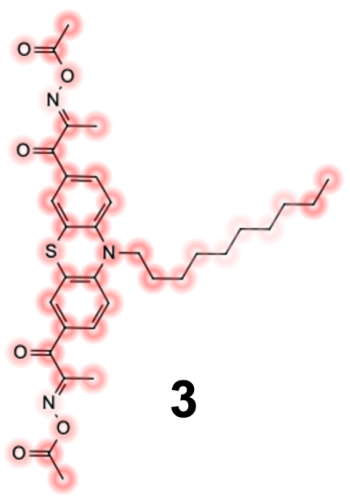

Figure S6. Model visualization for the three newly designed and synthesized photoinitiator candidates based on the best-performing continuous model.

\section{Method of synthesis for the new photoinitiator candidates}

The method of chemical synthesis for compound 1 is described below, along with an illustration of the 4step synthetic pathway (Scheme S1).

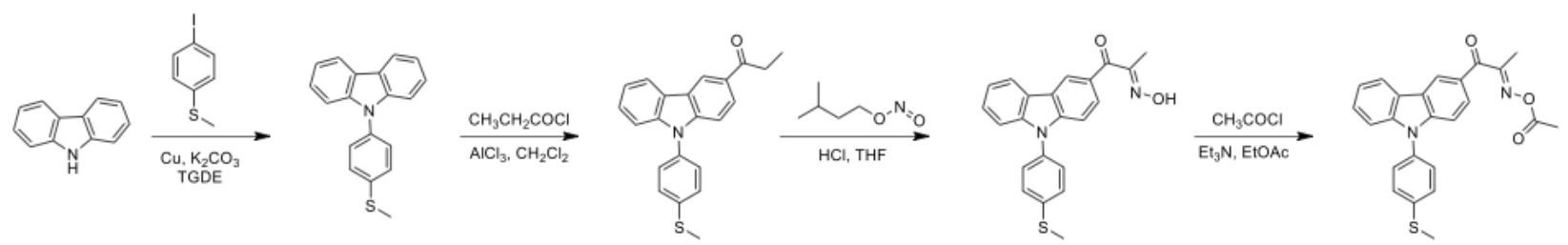

Scheme S1. Synthetic pathway for the newly-designed photoinitiator candidate 1.

Reaction 1: Synthesis of 9-(4-(methylthio)phenyl)-9H-carbazole

(a) $15.0 \mathrm{~g}$ of carbazole ( $85.2 \mathrm{mmol}), 25.7 \mathrm{~g}$ of 4 -iodothioanisole (99.68 mmol), $5.67 \mathrm{~g}$ of copper(powder) and $29.4 \mathrm{~g}$ of potassium carbonate $(211.6 \mathrm{mmol})$ are dissolved in $30 \mathrm{ml}$ of triethylene glycol dimethyl ether.

(b) The reaction mixture is stirred by mechanical at $200^{\circ} \mathrm{C}$ for 48 hours. 
(c) The reaction mixture is cooled to room temperature then $30 \mathrm{ml}$ of methylene chloride is added and stirred for 1 hour and filtered with silica gel pad.

(d) Filtrates are concentrated under reduced pressure to remove solvent.

(e) Residual crude products stand over one day at below $5^{\circ} \mathrm{C}$ to obtain precipitants.

(f) $200 \mathrm{ml}$ of ethanol was added then filtered.

(g) The product is recrystallized by ethanol to produce $22.5 \mathrm{~g}$ of 9-(4-(methylthio)phenyl)-9H-carbazole $(91.2 \%)$.

(f) Chemical structure is confirmed with NMR data: ${ }^{1} \mathrm{H}$ NMR $\left(\delta \mathrm{ppm} ; \mathrm{CDCl}_{3}\right): 2.60(3 \mathrm{H}, \mathrm{s}), 7.30(2 \mathrm{H}, \mathrm{t})$, $7.41(4 \mathrm{H}, \mathrm{m}), 7.48(4 \mathrm{H}, \mathrm{m}), 8.15(2 \mathrm{H}, \mathrm{d})$ as shown in Figure $\mathrm{S} 5$
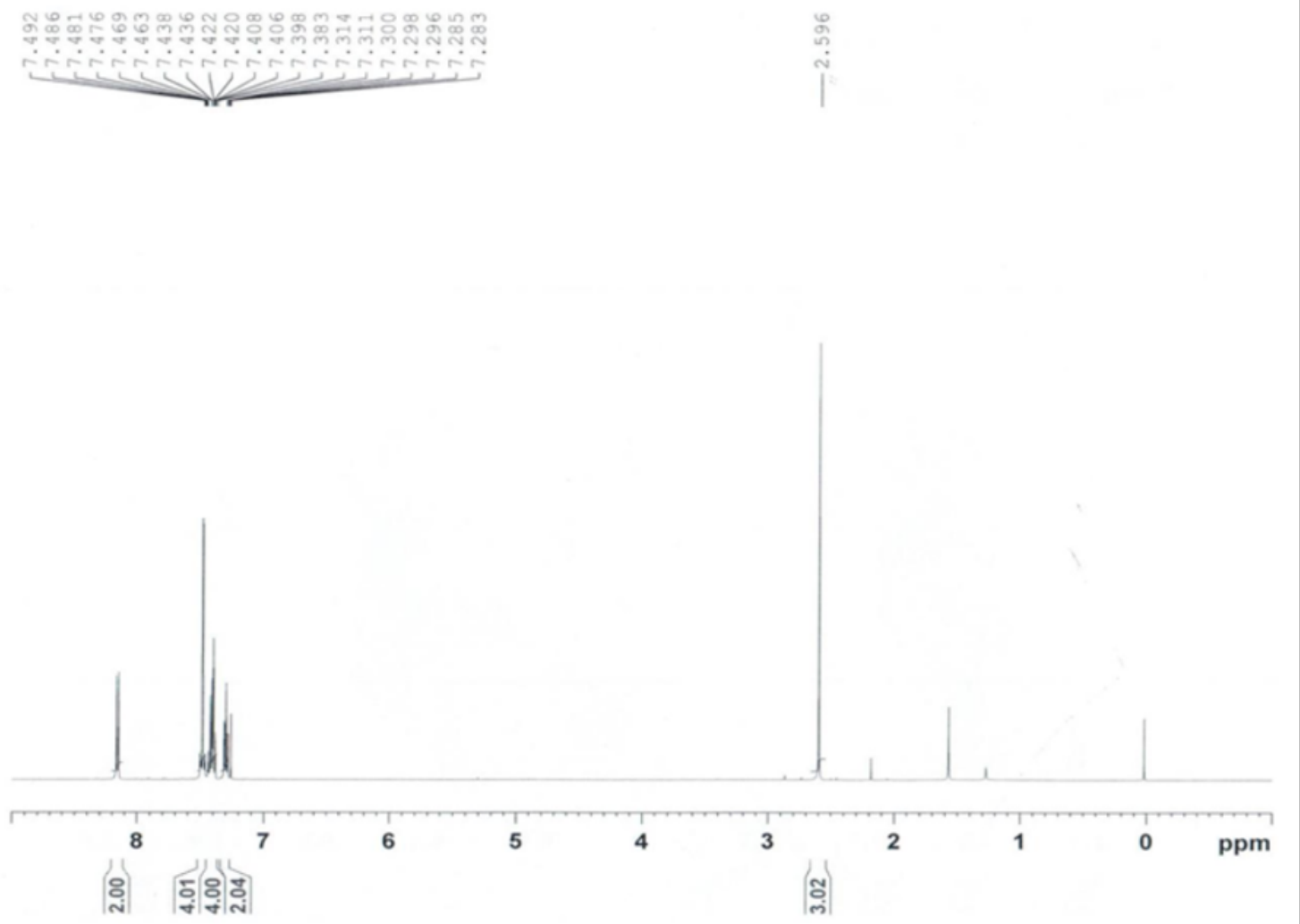

Figure S7. NMR data after reaction 1 for photoinitiator candidate 1.

Reaction 2: Synthesis of 1-(9-(4-(methylthio)phenyl)-9H-carbazol-3-yl)propan-1-one

(a) $2.8 \mathrm{~g}$ of aluminum chloride $(20.8 \mathrm{mmol})$ is added in $100 \mathrm{ml}$ of methylene chloride and stirred at $-10^{\circ} \mathrm{C}$ for 10

(b) $5 \mathrm{~g}$ of 9-(4-(methylthio)phenyl)-9H-carbazole $(17.3 \mathrm{mmol})$ is added and stirred for 30 minutes.

(c) $1.68 \mathrm{ml}$ of propionyl chloride is dissolved $10 \mathrm{ml}$ of methylene chloride then added to the reaction mixture and stirred for 30 minutes.

(d) The reaction mixture is poured into $50 \mathrm{~g}$ of ice and $200 \mathrm{ml}$ of water and stirred for 30 minutes.

(d) Separate the organic layer to wash with $100 \mathrm{ml}$ of saturated aqueous sodium bicarbonate solution and $100 \mathrm{ml}$ of distilled water. 
(e) Dry the organic layer over anhydrous magnesium sulfate and distill solvents under reduced pressure to produce $5.94 \mathrm{~g}$ of 1-(9-(4-(methylthio)phenyl)-9H-carbazol-3-yl)propan-1-one (99.4\%)

(f) Chemical structure is confirmed with NMR data: ${ }^{1} \mathrm{H}$ NMR $\left(\delta \mathrm{ppm} ; \mathrm{CDCl}_{3}\right): 1.31(3 \mathrm{H}, \mathrm{t}), 2.59(3 \mathrm{H}, \mathrm{s})$, $3.16(2 \mathrm{H}, \mathrm{q}), 7.35(3 \mathrm{H}, \mathrm{m}), 7.47(5 \mathrm{H}, \mathrm{m}), 8.07(1 \mathrm{H}, \mathrm{d}), 8.2(1 \mathrm{H}, \mathrm{d}), 8.8(1 \mathrm{H}, \mathrm{s})$ as shown in Figure $\mathrm{S} 6$.

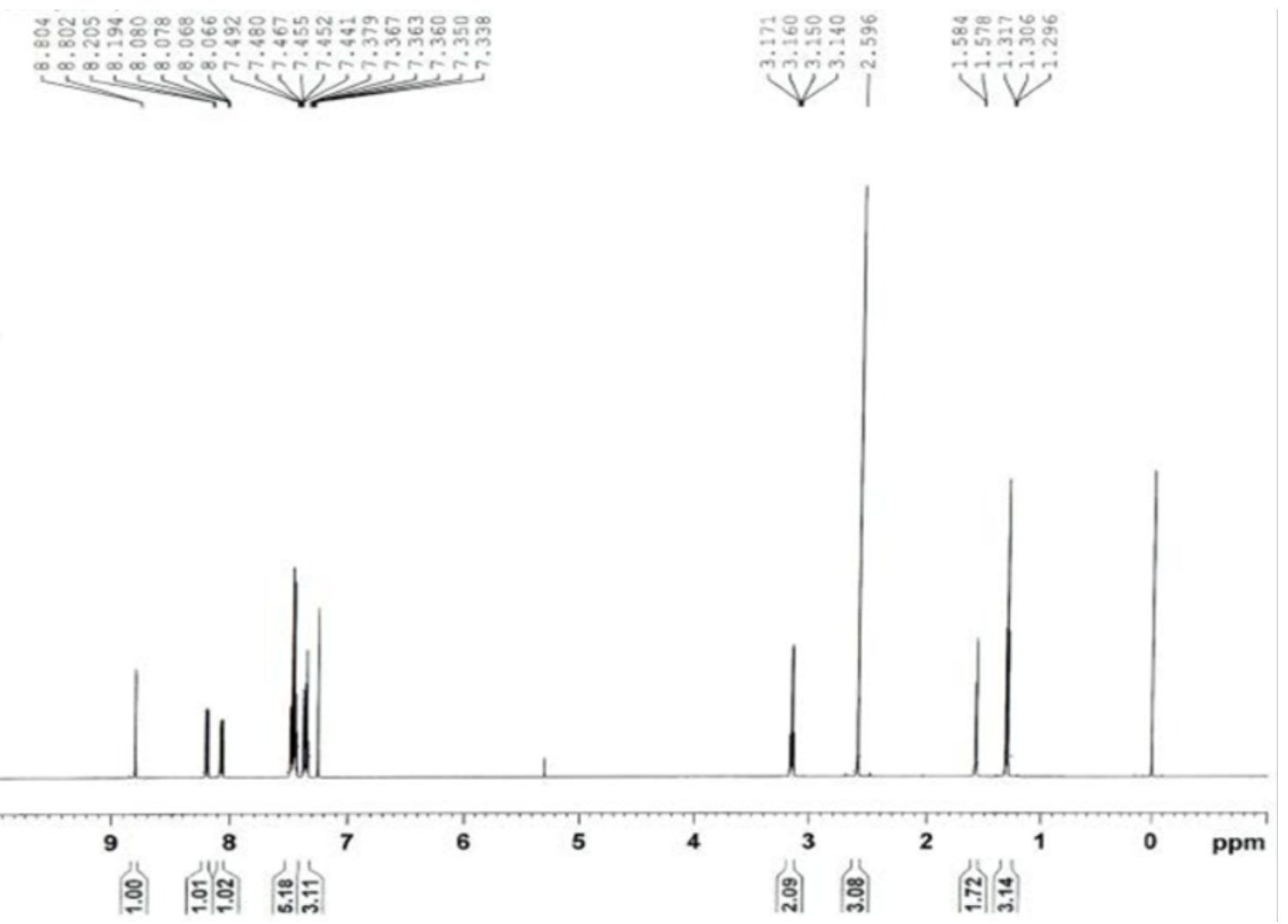

Figure S8. NMR data after reaction 2 for photoinitiator candidate 1.

Reaction 3: Synthesis of 1-(9-(4-(methylthio)phenyl)-9H-carbazol-3- yl)propan-1-one-2- oxime

(a) $5.8 \mathrm{~g}$ of 1-(9-(4-(methylthio)phenyl)-9H-carbazol-3-yl)propan-1-one $(16.8 \mathrm{mmol})$ and $3.2 \mathrm{ml}$ of concentrated hydrochloric acid $(37 \mathrm{mmol})$ are dissolved in $90 \mathrm{ml}$ of tetrahydrofuran and stirred for 20 minutes.

(b) $5.2 \mathrm{ml}$ of isopentyl nitrite $(37 \mathrm{mmol})$ in $10 \mathrm{ml}$ of tetrahydrofuran is added during 30 minutes and stirred for 50 minutes.

(c) $100 \mathrm{ml}$ of ethyl acetate is added and separated.

(d) Separate the organic layer to wash with $100 \mathrm{ml}$ of saturated aqueous sodium bicarbonate solution and $100 \mathrm{ml}$ of distilled water.

(e) Dry the organic layer over anhydrous magnesium sulfate and distill solvents under reduced pressure to produce crude product.

(f) The crude product is purified with hot filtration by methylene chloride to produce 2.9 of 1-(9-(4(methylthio)phenyl)-9H-carbazol-3- yl)propan-1-one-2- oxime (46.1\%)

(g) Chemical structure is confirmed with NMR data: ${ }^{1} \mathrm{H}$ NMR $\left(\delta \mathrm{ppm} ; \mathrm{CDCl}_{3}\right): 2.26(3 \mathrm{H}, \mathrm{s}), 2.59(3 \mathrm{H}, \mathrm{s})$, $7.35(3 \mathrm{H}, \mathrm{m}), 7.46(5 \mathrm{H}, \mathrm{m}), 7.9(1 \mathrm{H}, \mathrm{br}), 8.07(1 \mathrm{H}, \mathrm{d}), 8.18(1 \mathrm{H}, \mathrm{d}), 8.82(1 \mathrm{H}, \mathrm{s})$ as shown in Figure $\mathrm{S} 7$. 


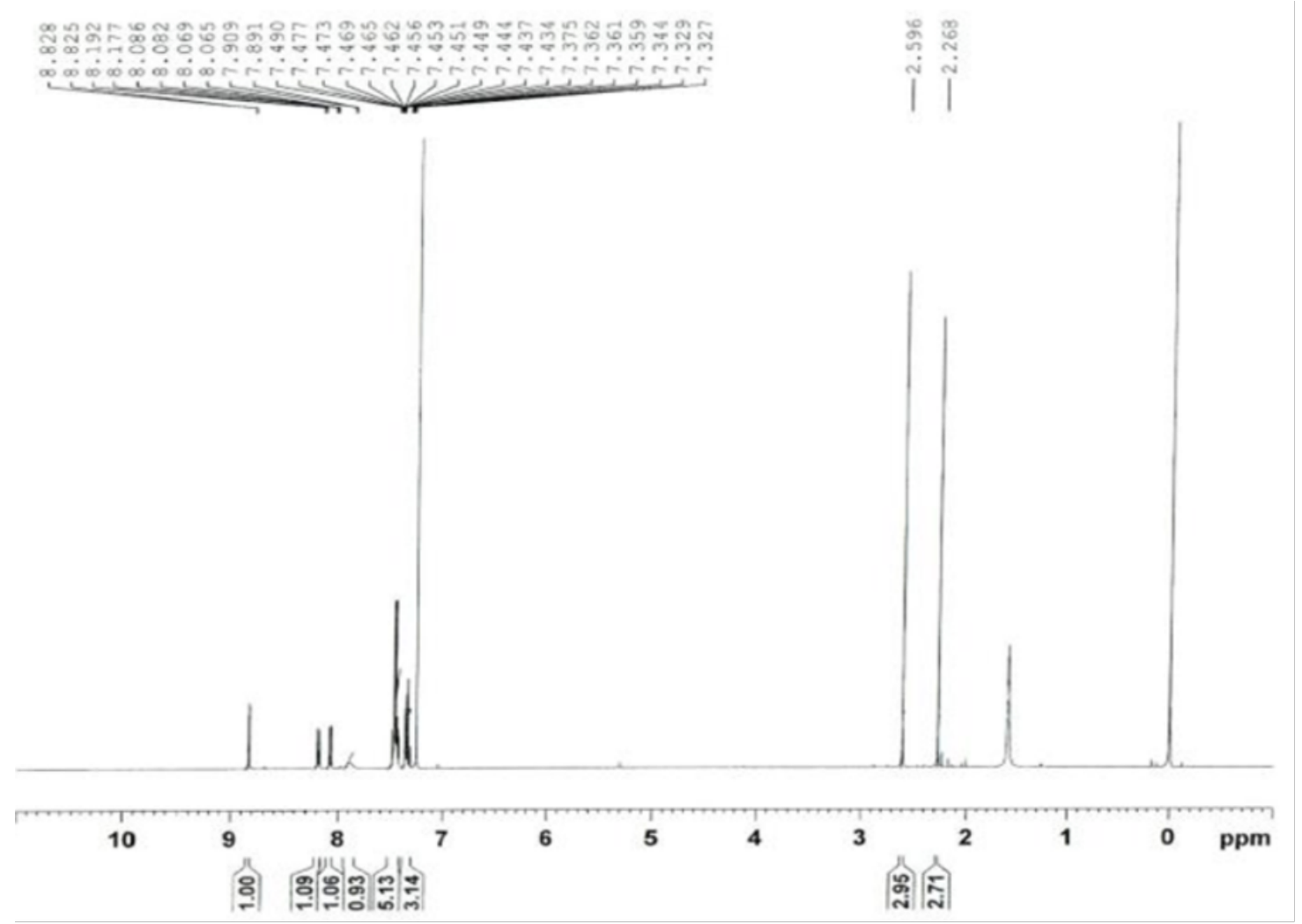

Figure S9. NMR data after reaction 3 for photoinitiator candidate 1.

Reaction 4: Synthesis of 1-(9-(4-(methylthio)phenyl)-9H-carbazol-3-yl)propan-1-one-2- oxime (O-acetate) (a) $1 \mathrm{~g}$ of 1-(9-(4-(methylthio)phenyl)-9H-carbazol-3- yl)propan-1-one-2- oxime (2.67 mmol) in $10 \mathrm{ml}$ of ethyl acetate and cooled to below $-10^{\circ} \mathrm{C}$ then added $0.75 \mathrm{ml}$ of trimethylamine $(5.34 \mathrm{mmol})$ and stirred for 10 minutes.

(b) $0.39 \mathrm{ml}$ of acetyl chloride $(5.34 \mathrm{mmol})$ in $1 \mathrm{ml}$ of ethyl acetate is added during 10 minutes and stirred for 20 minutes.

(c) Separate the organic layer to wash with $10 \mathrm{ml}$ of saturated aqueous sodium bicarbonate solution and $10 \mathrm{ml}$ of distilled water.

(d) Dry the organic layer over anhydrous magnesium sulfate and distill solvents under reduced pressure to produce crude product.

(f) The crude product is recrystallized by isopropyl alcohol to produce 0.92 of 1 -(9-(4-(methylthio)phenyl)$9 \mathrm{H}$-carbazol-3- yl)propan-1-one-2- oxime (O-acetate) (82.7\%)

(g) Chemical structure is confirmed with NMR data: ${ }^{1} \mathrm{H}$ NMR ( $\left.\delta \mathrm{ppm} ; \mathrm{CDCl}_{3}\right): 2.31(3 \mathrm{H}, \mathrm{s}), 2.37(3 \mathrm{H}, \mathrm{s})$, 2.59(3H, s), 7.36(3H, m), 7.47(5H, m), 8.2(2H, m), 8.99(1H, s) as shown in Figure S8. 

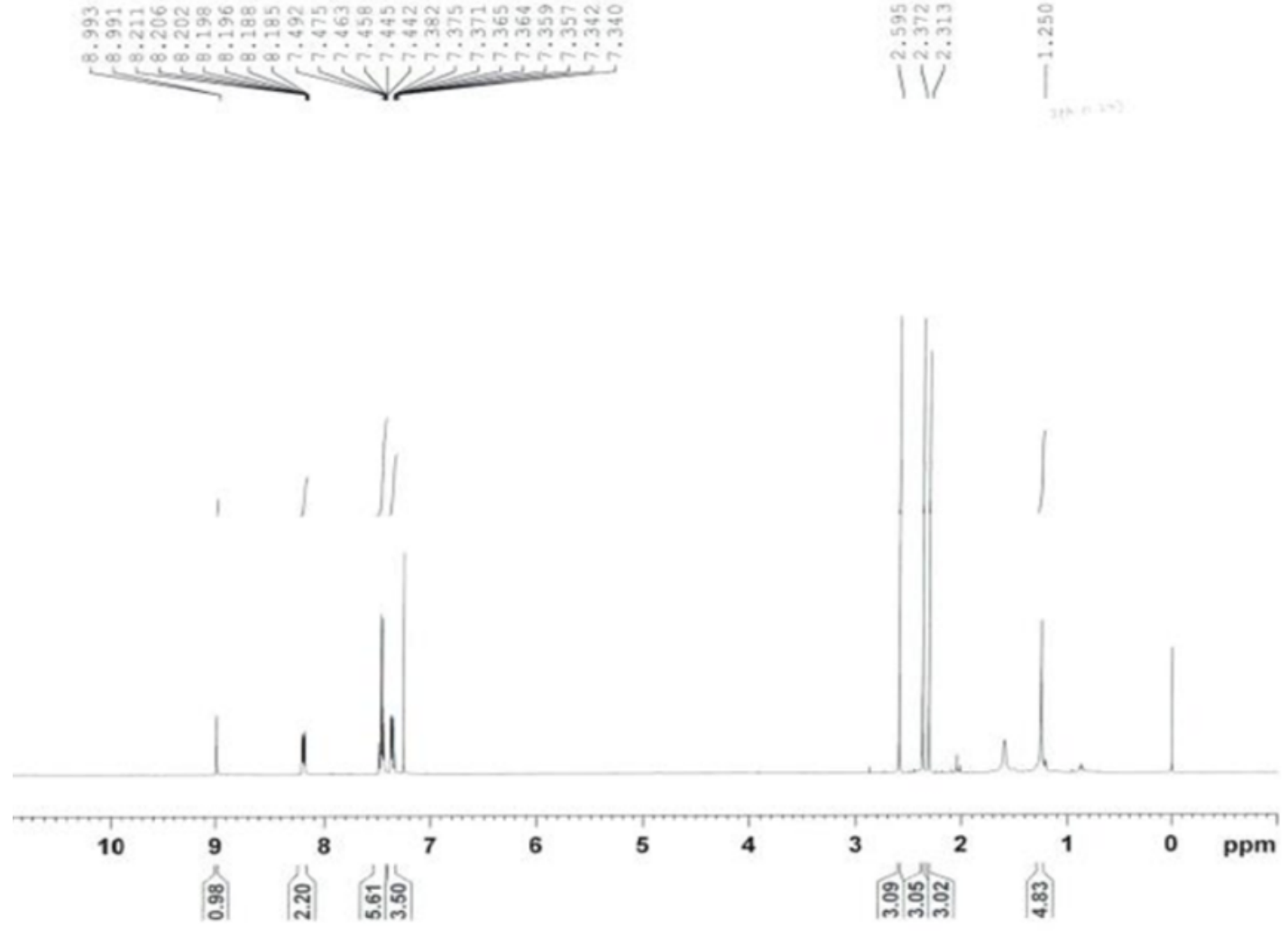

Figure S10. NMR data for photoinitiator candidate 1.

The method of chemical synthesis for compound $\mathbf{2}$ is described below, along with an illustration of the 4step synthetic pathway (Scheme S2).

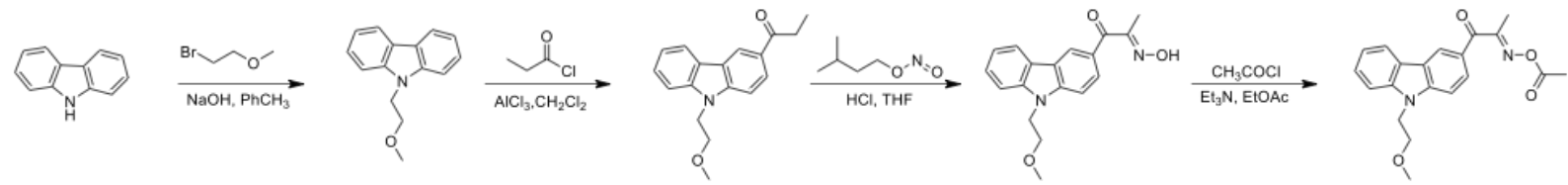

Scheme S2. Synthetic pathway for the newly-designed photoinitiator candidate 2.

Reaction 1: Synthesis of 9-(2-methoxyethyl)-9H-carbazole

(a) Dissolve $10.00 \mathrm{~g}$ of carbazole $(56.8 \mathrm{mmol}) 100 \mathrm{ml}$ of toluene and add $100 \mathrm{ml}$ of $50 \%$ aqueous $\mathrm{NaOH}$ solution .

(b) Add $17.1 \mathrm{ml}$ of bromoethyl methyl ether $(170.4 \mathrm{mmol})$ and stirred for 30 minutes.

(c) Add $1.0 \mathrm{ml}$ of tetrabutylammonium hydroxide solution, then stirred for 30 minutes at $70^{\circ} \mathrm{C}$.

(d) Add $70 \mathrm{ml}$ of distilled water and $50 \mathrm{ml}$ of ethyl acetate and stir for 30 minutes.

(e) Separate the organic layer and dry over anhydrous magnesium sulfate, and filter.

(f) Distilled under reduced pressure to remove solvent.

(g) Add ethanol and stirred to produce $11.80 \mathrm{~g}$ of 9-(2-methoxyethyl)-9H-carbazole $(87.4 \%)$.

(f) Chemical structure is confirmed with NMR data: ${ }^{1} \mathrm{H}$ NMR $\left(\delta \mathrm{ppm} ; \mathrm{CDCl}_{3}\right): 3.30(3 \mathrm{H}, \mathrm{s}), 3.77(2 \mathrm{H}, \mathrm{t})$, $4.49(2 \mathrm{H}, \mathrm{t}), 7.23(2 \mathrm{H}, \mathrm{m}), 7.46(4 \mathrm{H}, \mathrm{m}), 8.10(2 \mathrm{H}, \mathrm{d})$. 

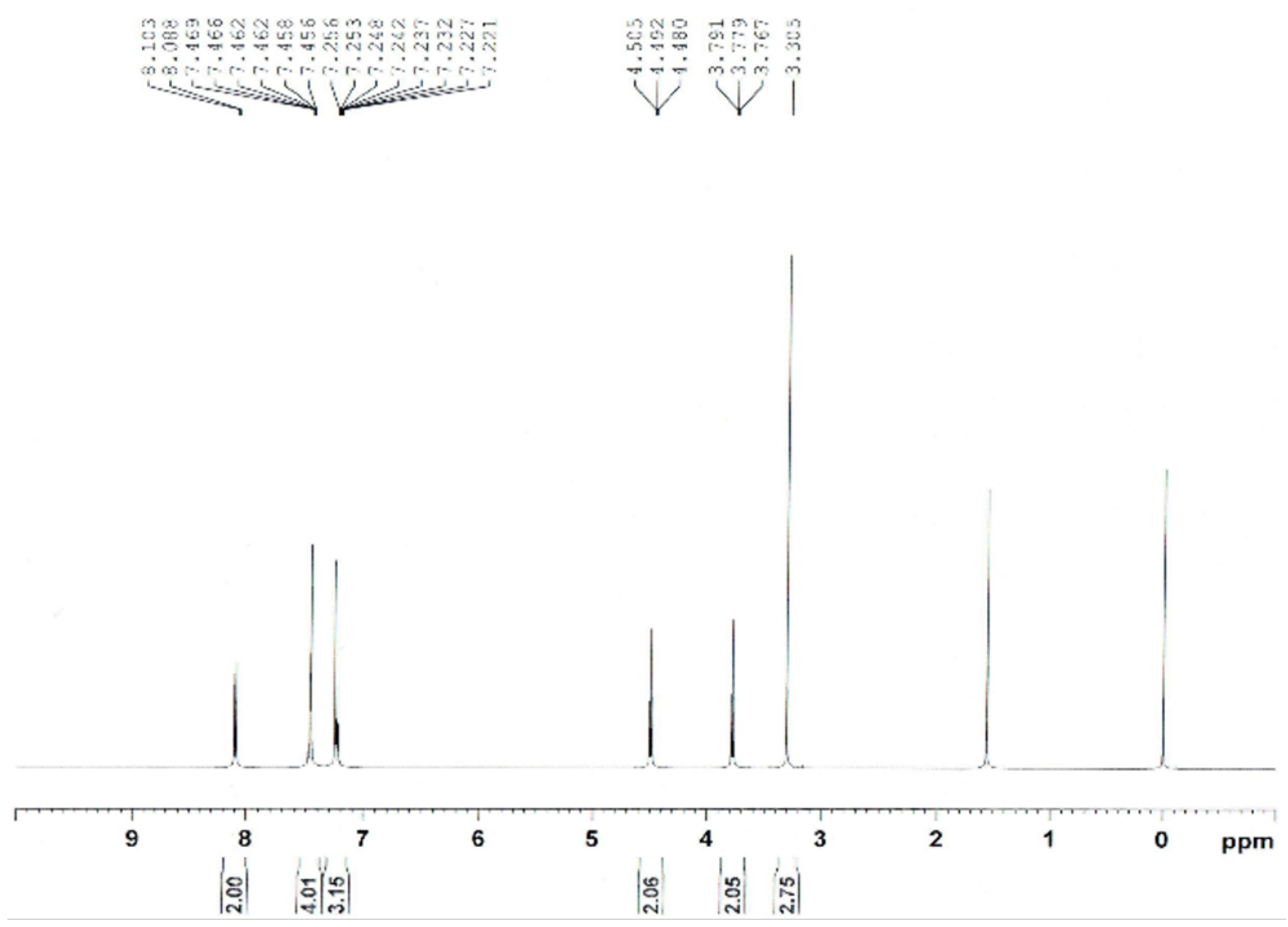

Figure S11. NMR data after reaction 1 for photoinitiator candidate 2.

Reaction 2: Synthesis of 1-(9-(2-methoxyethyl)-9H-carbazol-3-yl)propan-1-one

(a) Dissolve $5.98 \mathrm{~g}$ of anhydrous aluminum chloride $(44.4 \mathrm{mmol})$ in $50 \mathrm{ml}$ of dichloromethane and cool to $10^{\circ} \mathrm{C}$.

(b) Add $5.0 \mathrm{~g}$ of 9-(2-methoxyethyl)-9H-carbazole $(22.2 \mathrm{mmol})$ and stirred for 30 minutes.

(c) Add $3.95 \mathrm{ml}$ of propionyl chloride $(44.4 \mathrm{mmol}$ ) over 1 hour followed by stirring for another hour.

(c) Cool the mixture to room temperature, add $100 \mathrm{ml}$ of distilled water, stir for 30 minutes, and add $50 \mathrm{ml}$ of dichloromethane for extraction.

(d) Separate the organic layer to wash with $100 \mathrm{ml}$ of saturated aqueous sodium bicarbonate solution and $100 \mathrm{ml}$ of distilled water.

(e) Dry the organic layer over anhydrous magnesium sulfate and distill solvents under reduced pressure dispersed in a small amount of ethanol at room temperature.

(f) Filter and dry the solid product to obtain $5.05 \mathrm{~g}$ of 1-(9-(2-methoxyethyl)-9H-carbazol-3-yl)propan-1-one $(80.9 \%)$.

(g) Chemical structure is confirmed with NMR data: ${ }^{1} \mathrm{H}$ NMR $\left(\delta \mathrm{ppm} ; \mathrm{CDCl}_{3}\right): 1.30(3 \mathrm{H}, \mathrm{t}), 3.16(2 \mathrm{H}, \mathrm{q})$, $3.29(3 \mathrm{H}, \mathrm{s}), 3.79(2 \mathrm{H}, \mathrm{t}), 4.52(2 \mathrm{H}, \mathrm{t}), 7.31(1 \mathrm{H}, \mathrm{td}), 7.48(3 \mathrm{H}, \mathrm{m}), 8.15(2 \mathrm{H}, \mathrm{m}), 8.75(1 \mathrm{H}, \mathrm{sd})$. 

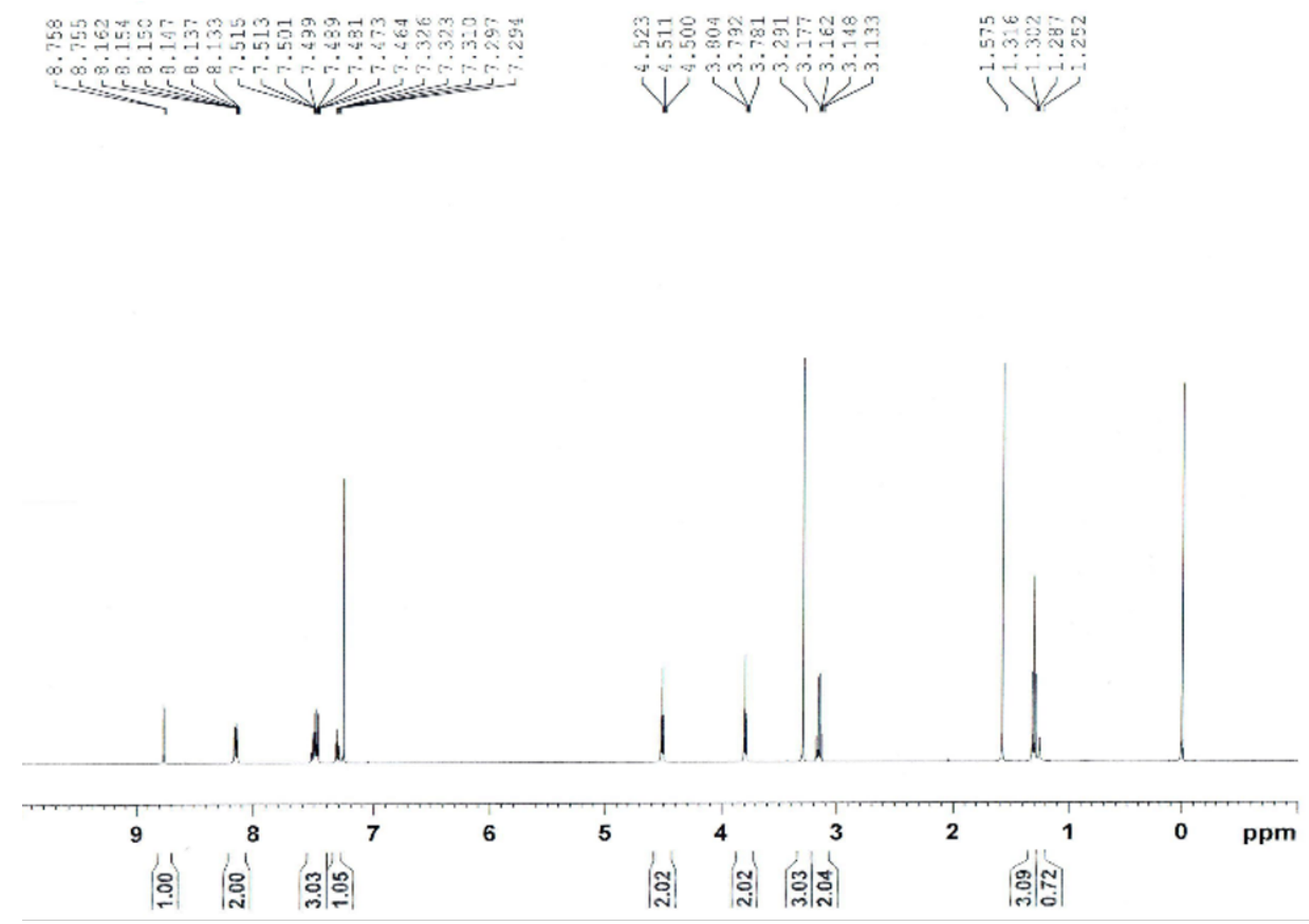

Figure S12. NMR data after reaction 2 for photoinitiator candidate 2.

Reaction 3: Synthesis of 1-(9-(2-methoxyethyl)-9H-carbazol-3-yl)propan-1-one-2-oxime

(a) Dissolve $3.00 \mathrm{~g}$ of 1-(9-(2-methoxyethyl)-9H-carbazol-3-yl)propan-1-one $(10.7 \mathrm{mmol})$ in $50 \mathrm{ml}$ of tetrahydrofuran and cool to $-10^{\circ} \mathrm{C}$.

(b) Add $1.89 \mathrm{ml}$ of hydrochloride solution $(15.1 \mathrm{mmol})$, and stirred for 30 minutes.

(c) Add $2.99 \mathrm{ml}$ of isopentyl nitrite $(21.4 \mathrm{mmol})$ and stirred for 3 hours at room temperature.

(d) Add $50 \mathrm{ml}$ of distilled water and stirred for 30 minutes, then add $50 \mathrm{ml}$ of ethyl acetate and stirred the mixture for 30 minutes.

(e) Separate the organic layer to wash with $100 \mathrm{ml}$ of saturated aqueous sodium bicarbonate solution and $100 \mathrm{ml}$ of distilled water.

(f) Dry the organic layer over anhydrous magnesium sulfate and distill solvents under reduced pressure to remove solvent.

(g) The product is purified using a silica gel column chromatography (eluent: ethyl acetate:n-hexane=1:2) to produce $1.92 \mathrm{~g}$ of 1-(9-(2-methoxyethyl)-9H-carbazol-3-yl)propan-1-one-2-oxime (58.0\%).

(h) Chemical structure is confirmed with NMR data: ${ }^{1} \mathrm{H}$ NMR $\left(\delta \mathrm{ppm} ; \mathrm{CDCl}_{3}\right) 2.26(3 \mathrm{H}, \mathrm{s}), 3.28(3 \mathrm{H}, \mathrm{s})$, 3.78(2H, t $), 4.02(2 \mathrm{H}, \mathrm{t}), 7.30(1 \mathrm{H}, \mathrm{td}), 7.47(3 \mathrm{H}, \mathrm{m}), 8.14(2 \mathrm{H}, \mathrm{m}), 8.77(1 \mathrm{H}, \mathrm{sd})$. 


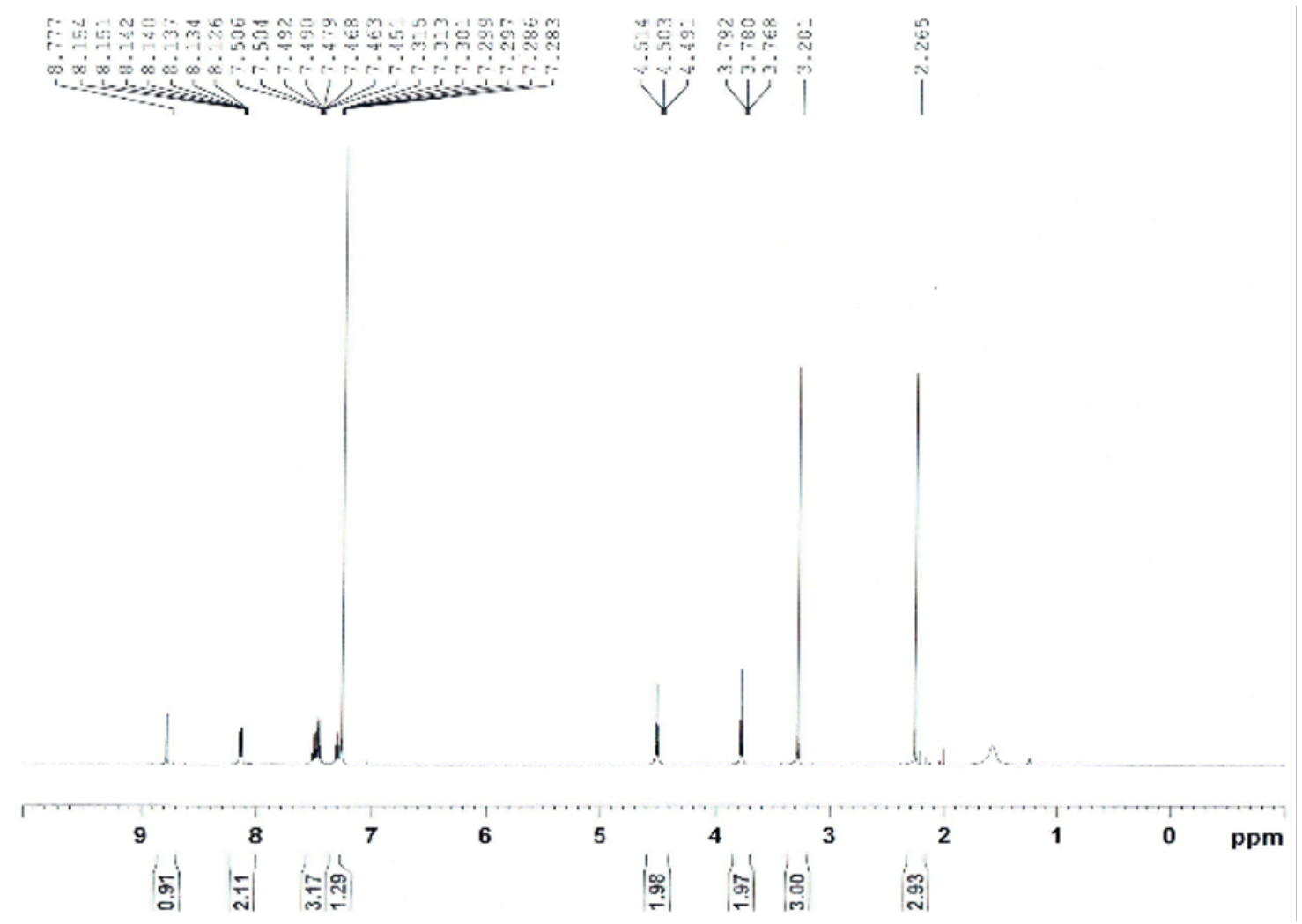

Figure S13. NMR data after reaction 3 for photoinitiator candidate 2.

Reaction 4: Synthesis of 1-(9-(2-methoxyethyl)-9H-carbazol-3-yl)propan-1-one-2-oxime -O-acetate

(a) Disperse $0.80 \mathrm{~g}$ of 1-(9-(2-methoxyethyl)-9H-carbazol-3-yl)propan-1-one-2-oxime (3.10 mmol) in $30 \mathrm{ml}$ of ethyl acetate and add $0.436 \mathrm{ml} \mathrm{g}$ of triethylamine $(3.10 \mathrm{mmol})$ and stirred for 30 minutes.

(b) Cool the reaction mixture to below $0^{\circ} \mathrm{C}$.

(c) Dissolve $0.224 \mathrm{ml}(3.10 \mathrm{mmol})$ of acetyl chloride in $1 \mathrm{ml}$ of ethyl acetate, drop it slowly, and stir.

(d) Add $10 \mathrm{ml}$ of distilled water, stir for 30 minutes, and add $10 \mathrm{ml}$ of ethyl acetate for extraction.

(e) Separate the organic layer to wash with $10 \mathrm{ml}$ of saturated aqueous sodium bicarbonate solution and $10 \mathrm{ml}$ of distilled water.

(f) Dry the organic layer over anhydrous magnesium sulfate and distill solvents under reduced pressure to obtain $0.90 \mathrm{~g}$ of 1-(9-(2-methoxyethyl)-9H-carbazol-3-yl)propan-1-one-2-oxime -O-acetate (99.1\%).

(d) Chemical structure is confirmed with NMR data: ${ }^{1} \mathrm{H}$ NMR $\left(\delta \mathrm{ppm} ; \mathrm{CDCl}_{3}\right): 2.31(3 \mathrm{H}, \mathrm{s}), 2.36(3 \mathrm{H}, \mathrm{s})$, $3.27(3 \mathrm{H}, \mathrm{s}), 3.78(2 \mathrm{H}, \mathrm{t}), 4.50(2 \mathrm{H}, \mathrm{t}), 7.31(1 \mathrm{H}, \mathrm{t}), 7.50(3 \mathrm{H}, \mathrm{m}), 8.14(1 \mathrm{H}, \mathrm{d}), 8.26(1 \mathrm{H}, \mathrm{dd}), 8.93(1 \mathrm{H}, \mathrm{s})$. 


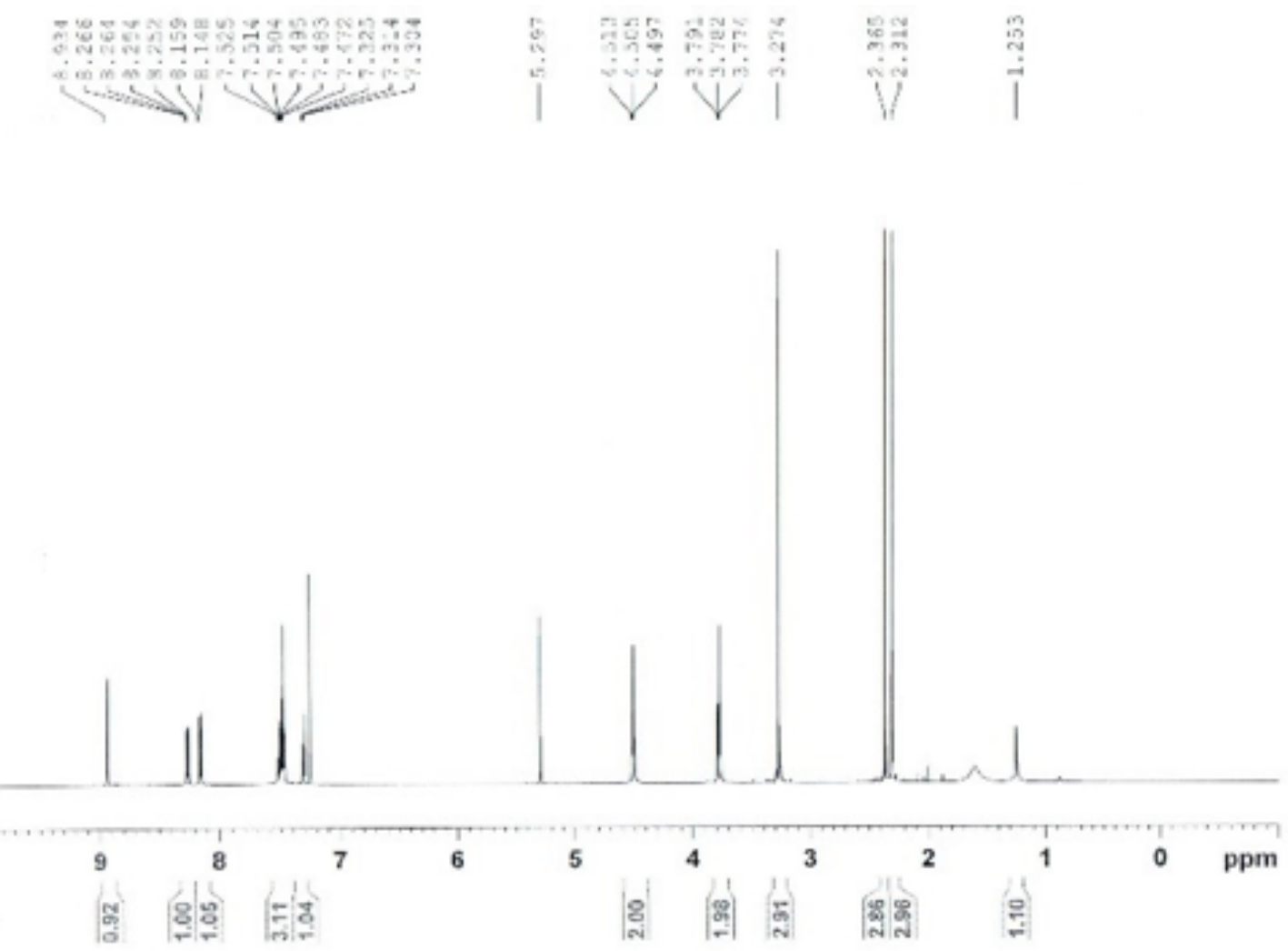

Figure S14. NMR data for photoinitiator candidate 2.

The method of chemical synthesis for compound 3 is described below, along with an illustration of the 4step synthetic pathway (Scheme S3).

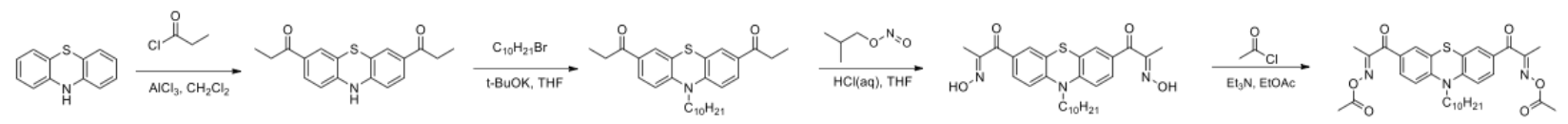

Scheme S3. Synthetic pathway for the newly-designed photoinitiator candidate 3.

Reaction 1: Synthesis of 1,1'-(10H-phenothiazine-3,7-diyl)bis(propan-1-one)

(a) Dissolve $100.0 \mathrm{~g}$ of phenothiazine $(0.5 \mathrm{~mol})$ in $500 \mathrm{ml}$ of dichloromethane and cool to below $10^{\circ} \mathrm{C}$.

(b) Add $160.59 \mathrm{~g}$ of anhydrous aluminum chloride $(1.20 \mathrm{~mol})$ and $111.0 \mathrm{~g}$ of propionyl chloride $(1.20 \mathrm{~mol})$.

(c) Warm to $25^{\circ} \mathrm{C}$ and stirred for 1 hour.

(d) Pour reaction mixture to $500 \mathrm{ml}$ iced distilled water and filtered.

(e) Separate the organic layer to wash with $500 \mathrm{ml}$ of saturated aqueous sodium bicarbonate solution and $500 \mathrm{ml}$ of distilled water.

(f) Dry the organic layer over anhydrous magnesium sulfate and distill solvents under reduced pressure to obtain 102.2g of 1,1'-(10H-phenothiazine-3,7-diyl)bis(propan-1-one) $(65.6 \%)$.

(g) Chemical structure is confirmed with NMR data: $1 \mathrm{H}$ NMR $(\delta \mathrm{ppm} ; \mathrm{CDCl} 3): 0.98(6 \mathrm{H}, \mathrm{t}), 2.81(4 \mathrm{H}, \mathrm{q})$, 6.52(2H, d), 7.53(2H, s), 7.61(2H, d). 


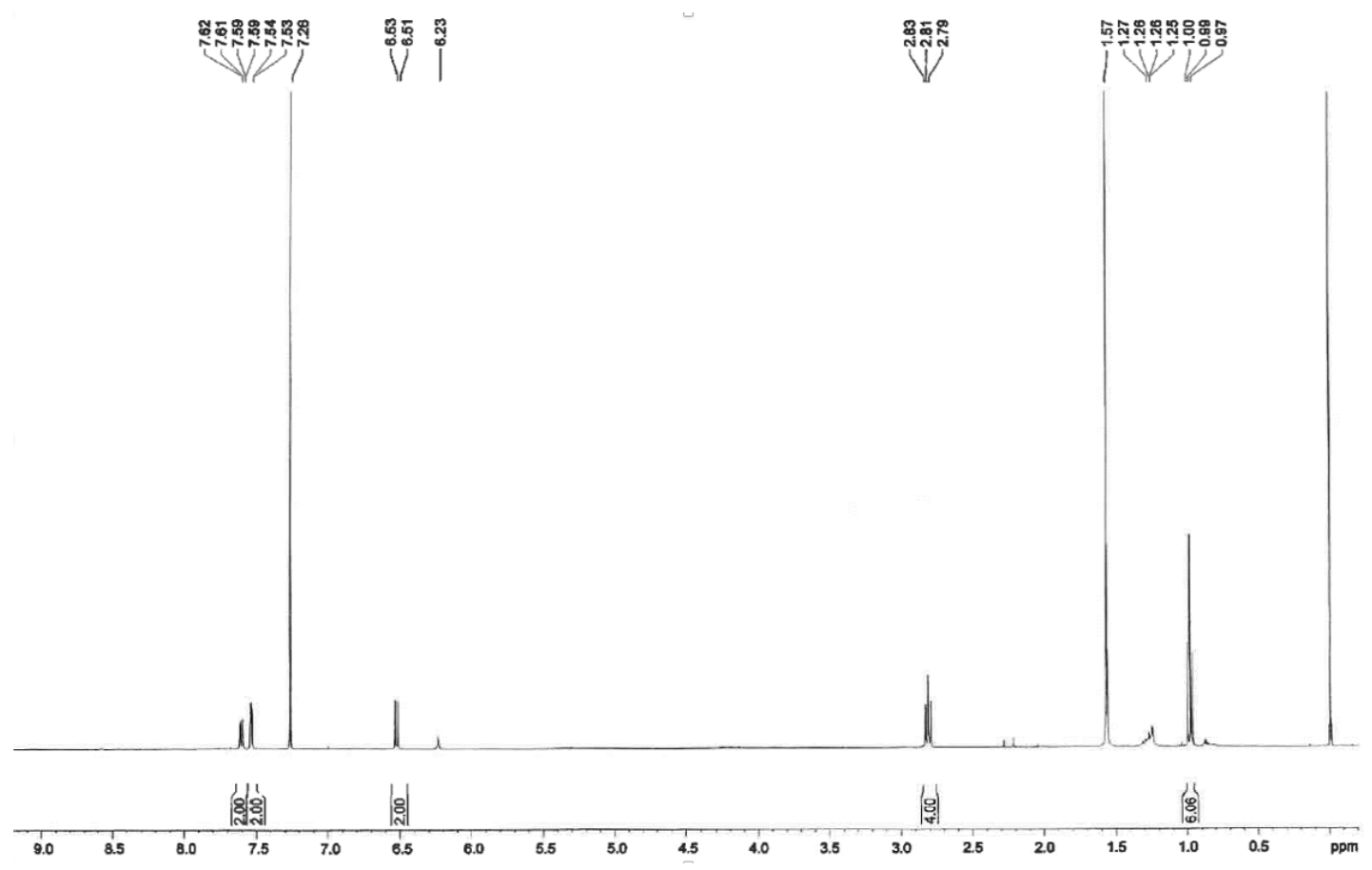

Figure S15. NMR data after reaction 1 for photoinitiator candidate 3.

Reaction 2: Synthesis of 1,1'-(10-decyl-10H-phenothiazine-3,7-diyl)bis(propan-1-one)

(a) Dissolve $90.3 \mathrm{~g}$ of 1,1'-(10H-phenothiazine-3,7-diyl)bis(propan-1-one) $(0.29 \mathrm{~mol})$ in $500 \mathrm{ml}$ of tetrahydrofuran and cool to $10^{\circ} \mathrm{C}$.

(b) Add $49.58 \mathrm{~g}$ of potassium tert-butoxide $(0.44 \mathrm{~mol})$ and add $130.32 \mathrm{~g}$ of bromodecane $(0.59 \mathrm{~mol})$ then stirred for 1 hour at $25^{\circ} \mathrm{C}$.

(c) Add 500 I of distilled water and stir for 30 minutes..

(d) Separate the organic layer to wash with $100 \mathrm{ml}$ of distilled water.

(e) Dry the organic layer over anhydrous magnesium sulfate and distill solvents under reduced pressure.

(f) Add ethanol and n-heptane to solidify.

(g) Filter and dry the solid product to obtain $120.12 \mathrm{~g}$ of 1,1'-(10-decyl-10H-phenothiazine-3,7-diyl) bis(propan-1-one) (91.7\%).

(g) Chemical structure is confirmed with NMR data: $1 \mathrm{H}$ NMR $(\delta \mathrm{ppm} ; \mathrm{CDCl} 3): 0.87(3 \mathrm{H}, \mathrm{t}), 0.99(6 \mathrm{H}, \mathrm{t}), 1.05-$ 1.48(16H, m), 1.70-1.83(2H, m), 2.85(4H, q), 3.89(2H, t), 6.86(2H, d), 7.68(2H, s), 7.76(2H, d). 


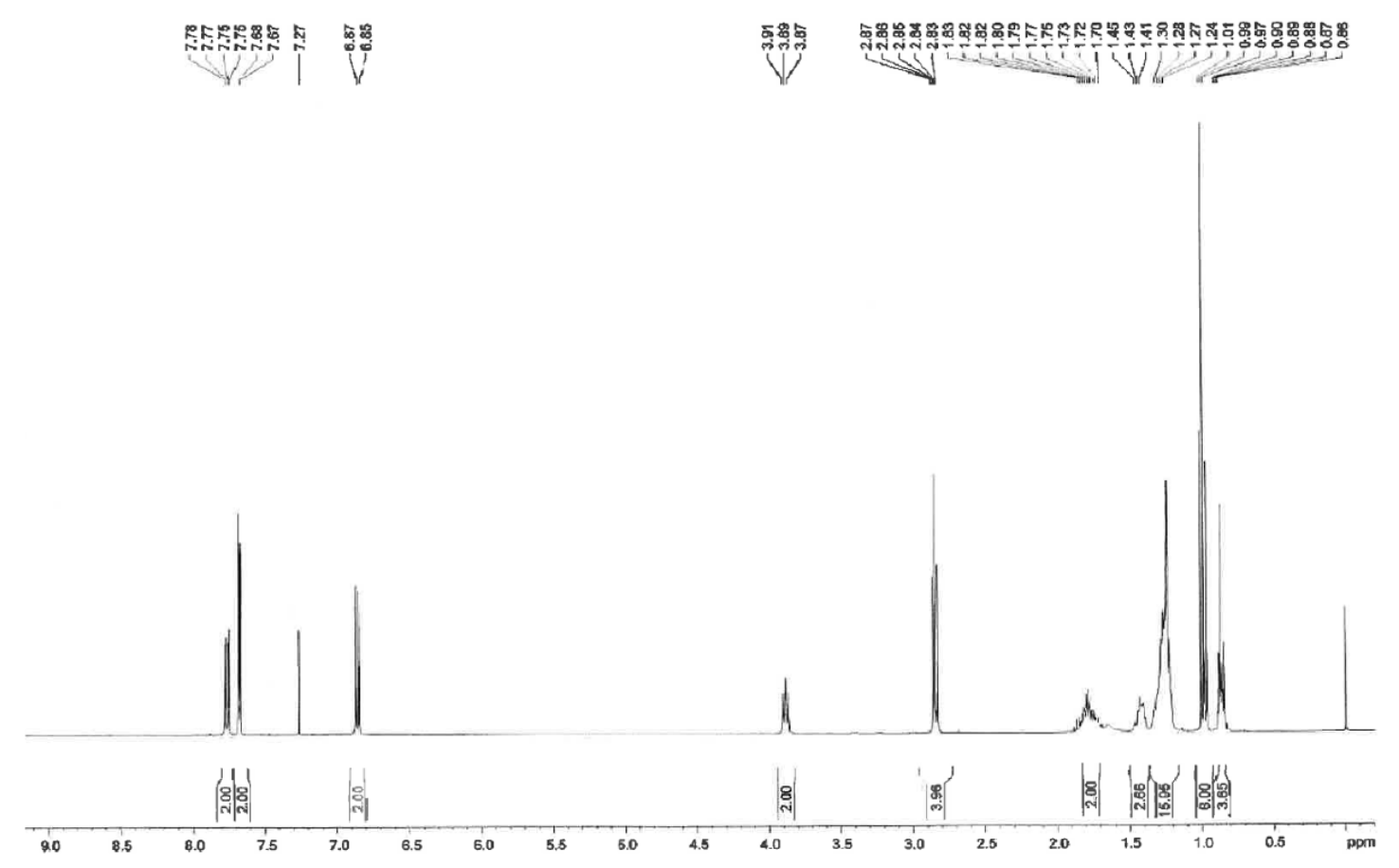

Figure S16. NMR data after reaction 2 for photoinitiator candidate 3.

Reaction 3: Synthesis of 1,1'-(10-decyl-10H-phenothiazine-3,7-diyl)bis(2-(hydroxyimino)propan-1-one) (a) Dissolve $94.9 \mathrm{~g}$ of 1,1'-(10-decyl-10H-phenothiazine-3,7-diyl)bis(propan-1-one) (0.21 mol) in $500 \mathrm{ml}$ of tetrahydrofuran and cool to $10^{\circ} \mathrm{C}$.

(b) Add $45.24 \mathrm{ml}$ of hydrochloride solution $(0.52 \mathrm{~mol})$, and stirred for 30 minutes.

(c) Add $53.74 \mathrm{~g}$ of isobutyl nitrite $(0.52 \mathrm{~mol})$ and stirred for 3 hours at $25^{\circ} \mathrm{C}$.

(d) Add $500 \mathrm{ml}$ of distilled water and stirred for 30 minutes, then add $500 \mathrm{ml}$ of ethyl acetate and stirred the mixture for 30 minutes.

(e) Separate the organic layer to wash with $100 \mathrm{ml}$ of saturated aqueous sodium bicarbonate solution and $100 \mathrm{ml}$ of distilled water.

(f) Dry the organic layer over anhydrous magnesium sulfate and distill solvents under reduced pressure to remove solvent.

(g) Add acetone and n-heptane to purify.

(h) Filter and dry the solid product to obtain $56.0 \mathrm{~g}$ of 1,1'-(10-decyl-10H-phenothiazine-3,7-diyl) bis(2(hydroxyimino)propan-1-one) (52.3\%).

(i) Chemical structure is confirmed with NMR data: $1 \mathrm{H} \mathrm{NMR}(\delta \mathrm{ppm} ; \mathrm{CDCl} 3) 0.91(3 \mathrm{H}, \mathrm{t}), 1.12(6 \mathrm{H}, \mathrm{t}), 1.21$ $1.58(16 \mathrm{H}, \mathrm{m}), 1.82-2.05(2 \mathrm{H}, \mathrm{m}), 4.24(2 \mathrm{H}, \mathrm{t}), 7.46(2 \mathrm{H}, \mathrm{d}), 8.31(2 \mathrm{H}, \mathrm{d}), 8.79(2 \mathrm{H}, \mathrm{s})$. 


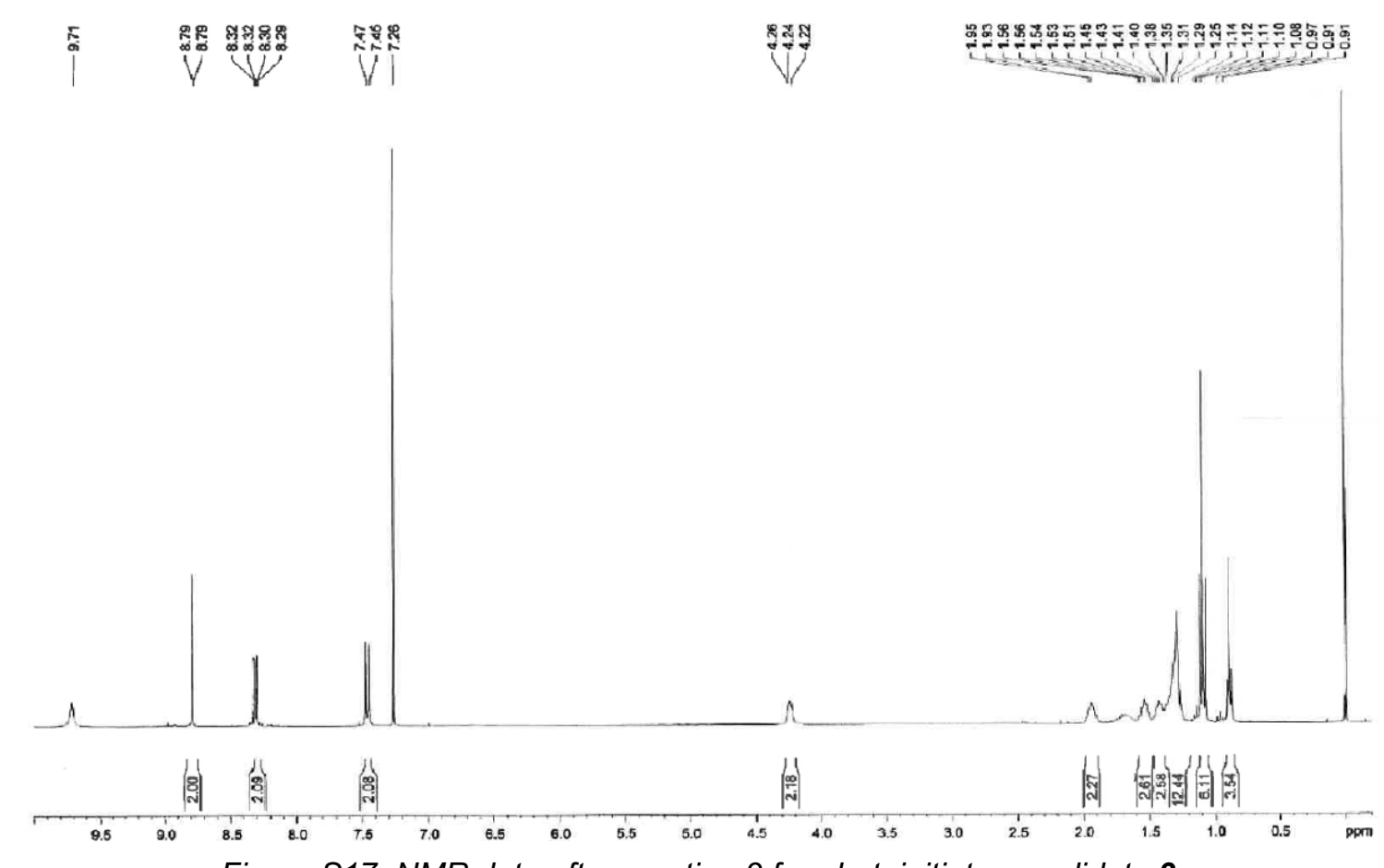

Figure S17. NMR data after reaction 3 for photoinitiator candidate 3.

Reaction 4: Synthesis of 1,1'-(10-decyl-10H-phenothiazine-3,7-diyl)bis(2-(acetoxyimino)propan-1-one)

(a) Disperse $45.9 \mathrm{~g}$ of 1,1'-(10-decyl-10H-phenothiazine-3,7-diyl)bis(2-(hydroxyimino)propan-1-one) (0.09 $\mathrm{mol})$ in $250 \mathrm{ml}$ of ethyl acetate and add $28.23 \mathrm{~g}$ of triethylamine $(0.28 \mathrm{~mol})$ and stirred for 30 minutes.

(b) Cool the reaction mixture to below $10^{\circ} \mathrm{C}$.

(c) Add $18.98 \mathrm{~g}$ of acetyl chloride $(0.24 \mathrm{~mol})$ and stirred for 1 hour at $25^{\circ} \mathrm{C}$.

(d) Add $250 \mathrm{ml}$ of distilled water, stir for 30 minutes.

(e) Separate the organic layer and dry the organic layer over anhydrous magnesium sulfate and distill solvents under reduced pressure.

(f) Add 2-propanol and $n$-heptane to solidified and purified with 2-propanol and isopropyl ether to obtain $34.6 \mathrm{~g}$ of 1,1'-(10-decyl-10H-phenothiazine-3,7-diyl)bis(2-(acetoxyimino)propan-1-one) $(64.7 \%)$.

(d) Chemical structure is confirmed with NMR data: $1 \mathrm{H} \mathrm{NMR}(\delta \mathrm{ppm} ; \mathrm{CDCl} 3): 0.91(3 \mathrm{H}, \mathrm{t}), 1.20(6 \mathrm{H}, \mathrm{t}), 1.21$ 1.60(16H, m), 1.90-2.05(2H, m), 2.31(6H, s), 4.31(2H, t), 7.53(2H, d), 8.48(2H, d), 8.82(2H, s). 


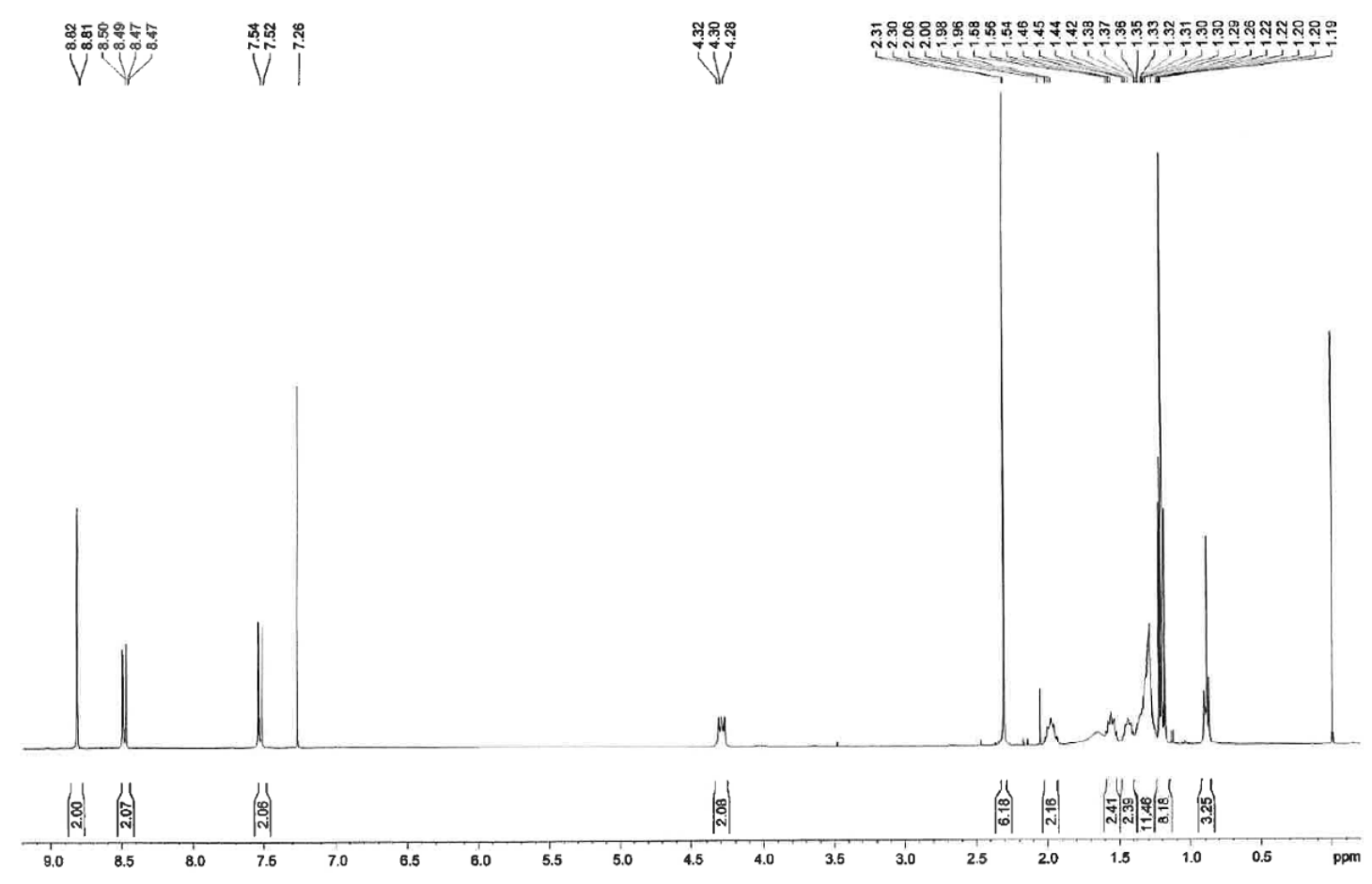

Figure S18. NMR data for photoinitiator candidate 3. 